\title{
Nonlinear dynamic response of a Negative Stiffness-Shape Memory Alloy isolation system
}

\section{Andrea Salvatore ( $\square$ a.salvatore@uniroma1.it )}

Universita degli Studi di Roma La Sapienza https://orcid.org/0000-0001-9718-9871

\section{Biagio Carboni}

Universita degli Studi di Roma La Sapienza

\section{Walter Lacarbonara}

Sapienza University of Rome: Universita degli Studi di Roma La Sapienza

\section{Research Article}

Keywords: High-static-low-dynamic stiffness isolator, Quasi-zero-stiffness isolation, Negative stiffness, Pseudo-elastic hysteresis

Posted Date: March 1st, 2021

DOl: https://doi.org/10.21203/rs.3.rs-186071/v1

License: (c) (1) This work is licensed under a Creative Commons Attribution 4.0 International License. Read Full License

Version of Record: A version of this preprint was published at Nonlinear Dynamics on July 18th, 2021. See the published version at https://doi.org/10.1007/s11071-021-06666-y. 


\title{
Nonlinear dynamic response of a Negative Stiffness-Shape Memory Alloy isolation system
}

\author{
Andrea Salvatore · Biagio Carboni · Walter \\ Lacarbonara
}

Received: date / Accepted: date

\begin{abstract}
The negative stiffness exhibited by bi-stable mechanisms together with tunable hysteresis in the context of vibration isolation devices can enhance the dynamic resilience of a structure. The effects of negative stiffness and shape memory alloy (SMA) damping in base-isolated structures are here explored by carrying out an extensive study of the nonlinear dynamic response via pathfollowing, bifurcation analysis, and time integration. The frequency-response curves of the isolated structure, with and without the negative stiffness contribution, are numerically obtained for different excitation amplitudes to construct the acceleration and displacement transmissibility curves. The advantages of negative stiffness, damping augmentation and reduced accelerations and displacements transmissibility, as well as the existence of rich bifurcation scenarios giving rise to quasi-periodicity and synchronization, are extensively illustrated.
\end{abstract}

Keywords High-static-low-dynamic stiffness isolator - Quasi-zero-stiffness isolation · Negative stiffness · Pseudo-elastic hysteresis

\section{Introduction}

Passive vibration isolation is one of the most popular and effective vibration control techniques. It is well known that for a SDOF oscillator, under harmonic forcing, there is a deamplification of the incoming accelerations for frequencies higher than $\sqrt{2} f_{0}$, where $f_{0}$ is the natural frequency of the oscillator. Therefore, the lower the natural frequency (i.e., the stiffness), the higher the isolated frequency bandwidth. On the other hand, an excessively low stiffness induces large static displacements. Hence, the need to obtain high static, low dynamic stiffness isolation systems. A very promising technique to achieve this goal is the exploitation of negative stiffness devices in parallel with classical vibration isolation devices.

Pasala, Nagarajaiah et al. [1,2] proposed and carried out analytical studies on an adaptive stiffness system with a negative stiffness component to simulate early yielding that can reduce seismic demands, whereby a bilinear spring is used in parallel with negative stiffness

A. Salvatore

Sapienza University of Rome

E-mail: a.salvatore@uniroma1.it 
in order to achieve an initial gap in the ensuing restoring force of the device. Other ways to achieve negative stiffness responses are the use of reverse bending sliding surfaces or magneto-rheological dampers with linear voltage decay [3 -9]. There are several developments of this concept applied to seismic isolation that show very promising results in terms of reduction of both accelerations and displacements transmissibilities in [10 -32], where a linear vertical isolation system is improved by introducing negative stiffness correction in order to achieve a high preload stiffness and a Quasi-Zero-Stiffness (QZS) in the equilibrium position. The nonlinear isolator response is usually described by a Duffing oscillator with a vanishing linear stiffness. Donmez et al. [33] studied the dynamic response of a dry-friction QZS isolator, showing that the hysteretic damping ensures a better performance than viscous damping in the out-of-resonance frequency range. In all of these works negative stiffness correction is used to achieve a zero stiffness in the equilibrium position, but as known, in a typical civil application dealing with seismic horizontal isolation, this is unwanted because of the need of a wind restraint. By delaying the negative stiffness contribution through an initial gap, the wind restraint is preserved, and transmissibility reduction can be achieved. Liu, Zhung et al. [34] proposed a novel isolation system composed by Shape Memory Alloy (SMA) wires and prestressed spring. The stiffness of SMA wires overcomes the negative stiffness exhibited by the prestressed spring until phase transformation occurs. For larger displacements, the stiffness of SMA wires vanishes and the overall stiffness becomes quasi zero. The transmissibility of a SDOF with the proposed response was analytically evaluated using a piece-wise linear constitutive law for the SMA response and a linear elastic law for the negative stiffness contribution. In the present work, the dynamic response of a classical seismic isolation system made up of elastomeric bearings working in parallel with a negative stiffness mechanism and SMA wires is explored. The SMA wires are used in order to realize the initial gap and, at the same time, provide hysteretic damping to the system while the negative stiffness contribution is used to achieve a zero dynamic stiffness away from the equilibrium position. The benefits associated with a negative stiffness mechanism together with hysteretic damping for an improved seismic isolation system are multifaceted. One of the main benefits is the possibility of reaching levels of deformability of the isolation layer beyond the limit represented by the deformability of the material constituting the isolation devices, obtaining quasi zero stiffness and ultra-low frequency isolation, hence substantial reduction in acceleration transmissibility otherwise not achievable through existing devices. Secondly, it is possible to leverage on the possibility of introducing high levels of hysteretic damping and amplifying the existing damping without loss of performance due to an increase in initial stiffness, given the fact that this increase is cancelled out by the appropriately tailored negative stiffness. For a careful investigation of the nonlinear stationary response of a SDOF representing the isolated structure controlled with a negative stiffnessSMA (NS-SMA) damping mechanism, both the nonlinear response of the bearing devices and the super-elastic response of SMA wires are modelled employing suitable hysteretic constitutive laws.

\section{Negative stiffness-SMA damper for seismic isolation}

In previous works, a bilinear spring is used in parallel with the negative stiffness in order to achieve an initial gap in the ensuing restoring force of the device. This gap allows to maintain for low amplitudes the virgin isolation stiffness and this is useful to realize a wind restraint. In this work, a super-elastic spring (Shape Memory Alloy wire) is used instead of the bilinear spring in order to realize the initial gap and, at the same time, deliver hysteretic 
damping to the system. The total restoring force $f$ of the proposed isolation system is thus the summation of the force $f_{i}$ provided by response of the traditional seismic elastomeric isolators, the force $f_{s}$ of SMA wires, and that provided by the negative stiffness mechanism denoted by $f_{n}$; that is,

$$
f=f_{i}+\left(f_{n}+f_{s}\right)=f_{i}+f_{n s}
$$

where $f_{n s}=f_{n}+f_{s}$ is the force of the proposed rheological device. For displacements below the gap, the stiffness of the wires is equal to the negative stiffness, hence, the overall response coincides with that of the elastomeric element. For displacements greater than the gap displacement, corresponding to the SMA wires yielding displacement, the negative stiffness strongly reduces the total force (base shear force) and stiffness. For larger displacements, the cubic term tends to overcome the negative stiffness contribution, thus making the overall response follow the baseline (elastomeric) backbone response (see Fig. 1).
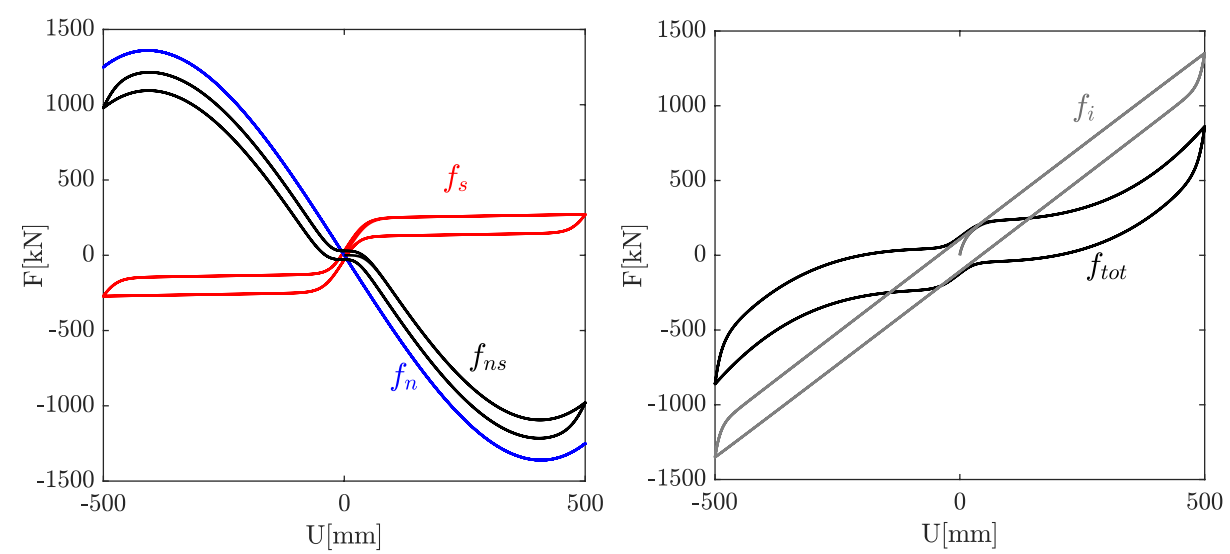

Fig. 1 Force-displacement cycles associated with (left) damper force $f_{n s}$ (black line) ensuing from $f_{s}$ (red line) plus $f_{n}$ (blue line) and with (right) the overall system response with and without damper (gray and black lines, respectively).

In the subsequent sections, 3 types of isolated SDOF systems are studied and compared. We conveniently identify them as follows: the baseline elastomeric isolation system (EIS) is referred to as $S_{1}$ and the associated restoring force is $f=f_{i}, S_{2}$ denotes the baseline isolation system together with the SMA damper alone and the associated restoring force is $f=f_{i}+f_{s}$ and, finally, $S_{3}$ indicates the proposed isolation system with the NS-SMA damper in parallel with the elastomeric bearings (see Fig. 2).

\section{$\mathbf{S}_{1}$ : Baseline isolation system}

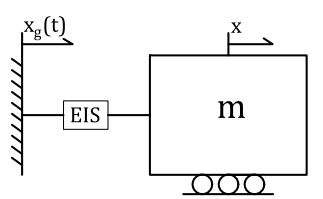

$\mathbf{S}_{2}$ : Baseline isolation system with SMA damper

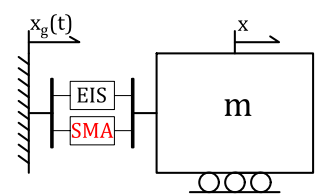

$\mathbf{S}_{\mathbf{3}}$ : Baseline isolation system with NS-SMA damper

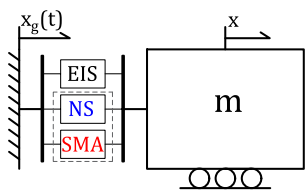

Fig. 2 Schematic representation of the 3 different isolated systems referred to as: $S_{1}, S_{2}$ and $S_{3}$. 
2.1 Rheological models

\subsubsection{Elastomeric isolators}

Elastomeric isolation systems are usually described by the Bouc-Wen model of hysteresis $[35,36]$ together with a linear viscous damping. The model is the direct summation of a viscous damping force, an elastic force and a hysteretic force. That is,

$$
f_{i}=c \dot{x}+\alpha K_{i} x+(1-\alpha) K_{i} z
$$

where the hysteretic force $\mathrm{z}$ is governed by

$$
\dot{z}=\dot{x}\left[1-(\gamma+\beta \operatorname{sign}(z \dot{x})]|z|^{n},\right.
$$

where $c$ is the viscous damping coefficient, $\alpha$ is the ratio between the post-elastic and the initial stiffness $K_{i}, \gamma$ and $\beta$ control the shape of the hysteresis loops, $n$ regulates the smoothness of transition between initial elastic and post-elastic stiffness. The upper and lower bounds of $z$ are given by $z_{m}=\sqrt[n]{(1-\alpha) K_{i} /(\gamma+\beta)}$. In the present study, $\gamma+\beta$ is restricted to be positive in order to have a softening behaviour and $n$ is set to 1 (Fig. 3).

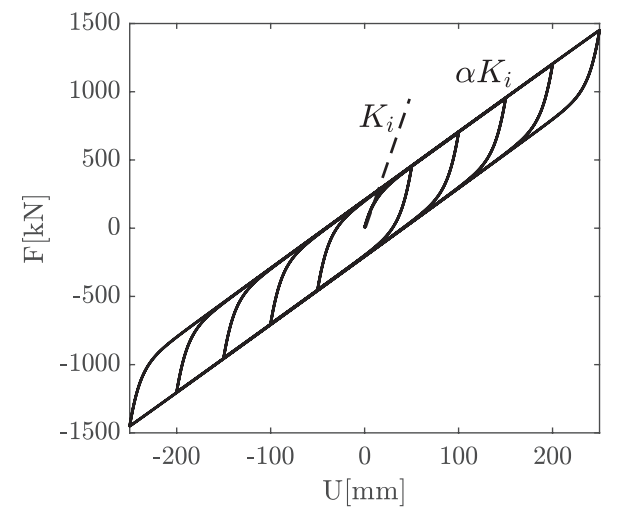

\begin{tabular}{cc}
\multicolumn{2}{c}{ Isolation system parameters } \\
\hline$K_{i}$ & Initial stiffness \\
\hline$\alpha$ & 0.2 \\
\hline$\gamma$ & 0.01 \\
\hline$\beta$ & 0.09 \\
\hline$n$ & 1 \\
\hline$c$ & 0.1 \\
\hline
\end{tabular}

Fig. 3 Force-displacement cycles of isolation devices (left) and model parameters (right)

\subsubsection{Negative stiffness mechanism}

In most passive bi-stable mechanisms, the negative stiffness is produced by geometric nonlinearities that cause negative stiffness only in a given displacement range and positive stiffness outside. In this work the positive force exhibited past the displacement $x_{f}$, where the force vanishes, is cancelled by means of a step function. The NS force reads

$$
f_{n}=\left(-K_{n} x+K_{3} x^{3}\right) \frac{\left(1+\operatorname{sign}\left(x_{f}-|x|\right)\right)}{2}
$$

where $K_{n}$ is the negative linear stiffness, $K_{3}$ is the positive cubic stiffness, and $x_{f}$ is the displacement corresponding to a vanishing force and is equal to $x_{f}=\sqrt{K_{n} / K_{3}}$. Another characteristic displacement is the displacement where the maximum negative force is reached given by $x_{n}=\sqrt{K_{n} / 3 K_{3}}$ (see Fig.4). 


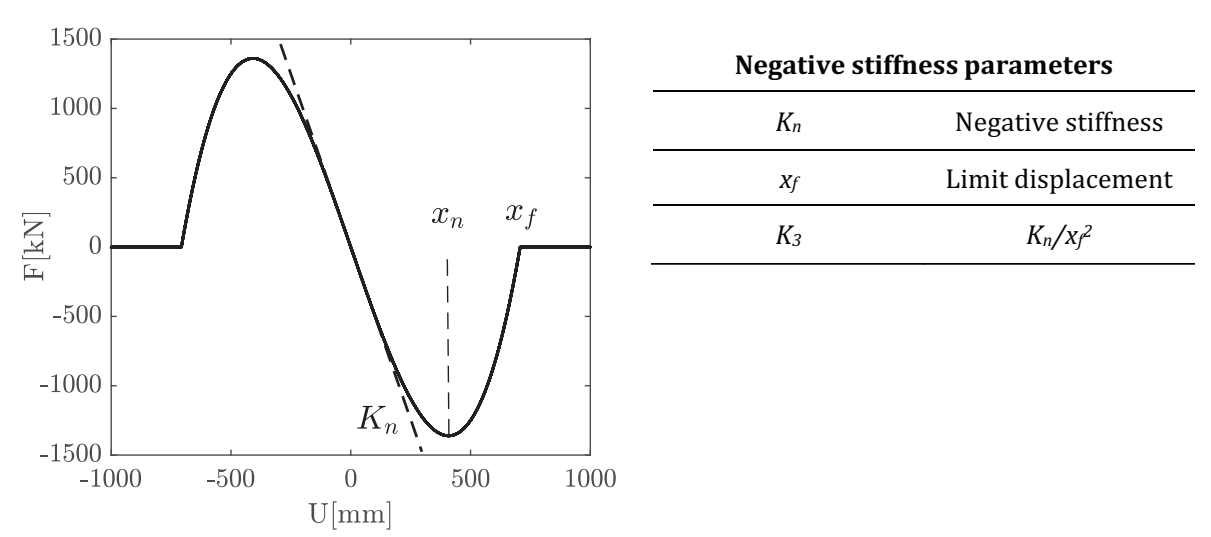

Fig. 4 Force-displacement cycles provided by the negative stiffness force (left) and model parameters (right).

\subsubsection{SMA wires}

The response of SMA wires is modelled according to the phenomenological super-elastic hysteretic model proposed by Charalampakis [37] given in rate form:

$$
\begin{aligned}
& \dot{f}_{s}=(1-s) K_{s}\left[\dot{x}-|\dot{x}| \operatorname{sign}\left(f_{s}-\beta_{s}\right)\left(\frac{\left|f_{s}-\beta_{s}\right|}{Y}\right)^{n_{s}}\right]+s K_{m} \dot{x}, \\
& \beta_{s}=K_{s} \alpha_{s}\left[x-\frac{f_{s}}{K_{s}}+f_{t} \tanh \left(a_{s} x\right)\left[\frac{1+\operatorname{sign}(-x \dot{x})}{2}\right]\right], \\
& s=\frac{\tanh \left[c_{s}\left(|x|-x_{m}\right)\right]+1}{2},
\end{aligned}
$$

where $K_{s}$ is the initial stiffness during the austenitic phase, $Y$ is the yielding force, $\alpha_{s}$ controls the post-elastic stiffness, $n_{s}$ regulates the smoothness of transition from the initial elastic to the post-elastic phase, $f_{t}$ controls the twinning hysteresis and super-elasticity, $a_{s}$ governs the pinching around the origin during cyclic loading, $K_{m}$ is the stiffness during the fully martensitic phase, $x_{m}$ is the displacement at which the transition from the post-elastic to the fully martensitic phase occurs, while $c_{s}$ control the smoothness of this transition (see Fig. $5)$.

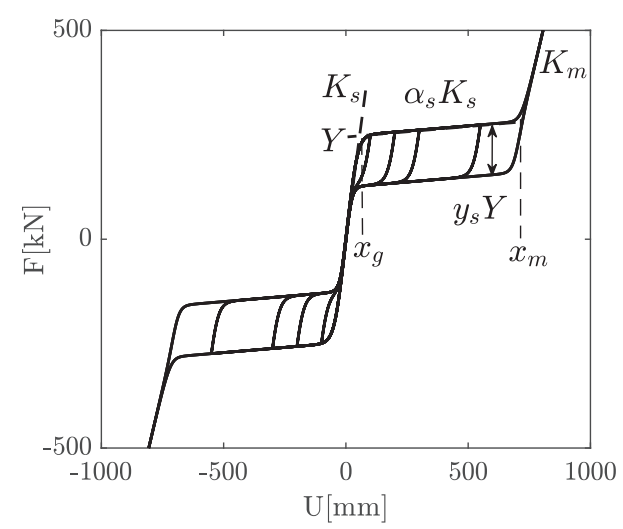

\begin{tabular}{cc}
\multicolumn{2}{c}{ SMA wires parameters } \\
\hline$Y$ & Yielding force \\
\hline$x_{g}$ & Gap displ. \\
\hline$K_{s}$ & $Y / x_{g}$ \\
\hline$\alpha_{s}$ & 0.01 \\
\hline$f_{t}$ & 6000 \\
\hline$n_{s}$ & 3 \\
\hline$a_{s}$ & 0.06 \\
\hline$K_{m}$ & $0.5 K_{s}$ \\
\hline$c_{s}$ & 0.02 \\
\hline$x_{m}$ & Martensitic displ. \\
\hline
\end{tabular}

Fig. 5 Force-displacement cycles of the SMA wires (left) and model parameters (right). 
Because of the large variability and dependence of parameters $\mathrm{ft}$ and as on the remaining parameters, two new parameters with a more straightforward physical interpretation, $y_{s}$ and $\tilde{a}_{s}$, are introduced. In terms of these new parameters, $f_{t}$ and $a_{s}$ are expressed as:

$$
\begin{gathered}
f_{t}=\left(2 Y-y_{s} Y\right) /\left(\alpha_{s} K_{s}\right), \\
a_{s}=\tanh ^{-1}\left(\tilde{a}_{s} K_{s}\right) /\left(Y-y_{s} Y\right) .
\end{gathered}
$$

The advantage of introducing these new parameters is associated with the possibility of obtaining the same cycles by changing a parameter of interest, useful for parametric analyses. The first parameter indicates the difference between the loading and unloading forces expressed as percentage of $Y$, the second allows has been reformulated in order to obtain, with the same coefficient, the same residual displacement, and therefore the same hysteresis loop shape, for different values of the other parameters (see Fig. 6). In particular, $\tilde{a}_{s}$ indicates the value assumed by $\tanh \left(\tilde{a}_{s} x\right)$ for a displacement $x$ at which the continuation of the unloading branch intersects the elastic loading branch.
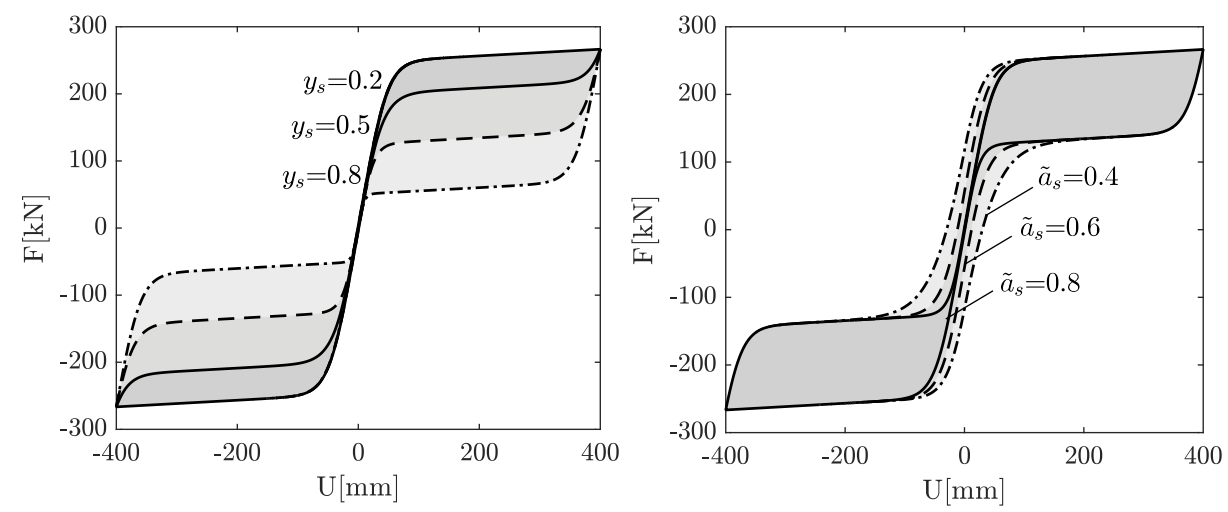

Fig. 6 Variation of the force-displacement cycles of SMA wires with the nondimensional parameters $y_{s}$ (left) and $\tilde{a}_{s}$ (right).

\subsubsection{Design Parameters}

The main goal is to investigate the effects of the NS-SMA device in parallel with a traditional elastomeric device as a hysteretic isolation system. Hence, the stiffness $K_{i}$ of the isolation system, the gap displacement $x_{g}=F_{w} / K_{i}$, (with $F_{w}$ the maximum expected wind load) and the maximum allowed displacement $x_{u}$ are assumed as input parameters (see Fig. 7). In this study as reasonable value of $x_{g}$ is selected $x_{g}=0.05 x_{u}$. On the other hand, $K_{n}, Y$ and $y_{s}$ are the design parameters. All the remaining parameters are either set on fixed values $\left(c=0.1, \alpha=0.2, \beta=0.09, \gamma=0.01, n=1, \alpha_{s}=0.01, n_{s}=3, \tilde{a}_{s}=0.6, c_{s}=0.02\right)$ or determined accordingly $x_{f}=x_{m}=0.7 x_{u}, K_{3}=K_{n} / x_{f}^{2}, K_{s}=Y / x_{g}, f_{t}=\left(2 Y-y_{s} Y\right) /\left(\alpha_{s} K_{s}\right)$, $a_{s}=\tanh ^{-1}\left(\tilde{a}_{s} K_{s}\right) /\left(Y-y_{s} Y\right), K_{m}=0.5 K_{s}$. 


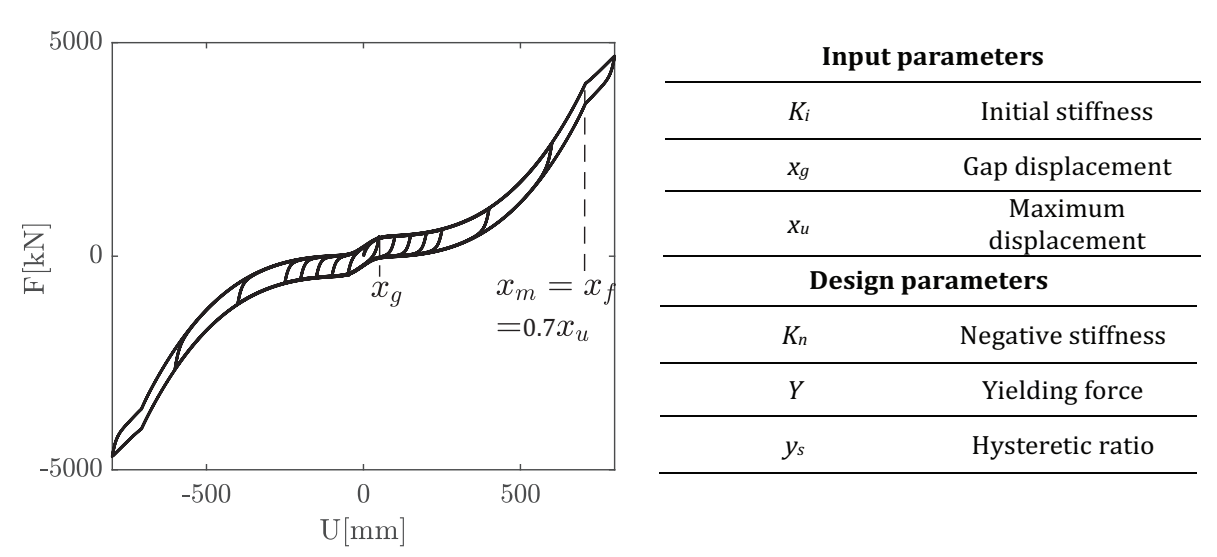

Fig. 7 Overall force-displacement cycles provided by $S_{3}$ (left) and system parameters (right)

\subsubsection{Nondimensional equation of motion}

The equation of motion of a SDOF mass m subject to the hysteretic restoring force given by Eq. (1) reads:

$$
m \ddot{x}+c \dot{x}+\alpha K_{i} x+(1-\alpha) K_{i} z+\left(-K_{n} x+K_{3} x^{3}\right) \frac{\left(1+\operatorname{sign}\left(x_{f}-|x|\right)\right)}{2}+f_{s}=P \sin \left(\Omega_{g} t\right) .
$$

By choosing a characteristic displacement $x_{0}=x_{u}$ and a characteristic stiffness $K_{i}$ (i.e., the initial stiffness of the isolation system, equal to the initial stiffness of the Bouc-Wen model), the following nondimensional variables are introduced $\tilde{x}=x / x_{0}, \tilde{t}=\omega t$, where $\omega=\sqrt{K_{i} / m}$ while the characteristic force is $N_{0}=K_{i} x_{0}$. By dividing the equation of motion by $N_{0}$, the following nondimensional equation of motion is obtained:

$$
\ddot{\tilde{x}}+\zeta \dot{\tilde{x}}+\alpha \tilde{x}+(1-\alpha) \tilde{z}+\left(-\tilde{K}_{n} \tilde{x}+\tilde{K}_{3} \tilde{x}^{3}\right) \frac{\left(1+\operatorname{sign}\left(\tilde{x}_{f}-|\tilde{x}|\right)\right)}{2}+\tilde{f}_{s}=\tilde{P}_{\sin }\left(\tilde{\Omega}_{g} \tilde{t}\right),
$$

with $\tilde{P}=P / N_{0}, \tilde{\Omega}_{g}=\Omega_{g} / \omega$. Consequently, the nondimensional elastomeric isolators force becomes

$$
\tilde{f}_{i}=\frac{f_{i}}{N_{0}}=\zeta \dot{\tilde{x}}+\alpha \tilde{x}+(1-\alpha) \tilde{z}
$$

where $\zeta=c \omega / K_{i}$. Equation (3) can be rewritten in nondimensional form as

$$
\dot{\tilde{z}}=\frac{\dot{z}}{x_{0} \omega}=\dot{\tilde{x}}\left[1-(\tilde{\gamma}+\tilde{\beta} \operatorname{sign}(\tilde{z} \dot{\tilde{x}}))|\tilde{z}|^{n}\right],
$$

where $\tilde{z}=\frac{z}{x_{0}}, \tilde{\gamma}=\gamma x_{0}^{n}, \tilde{\beta}=\beta x_{0}^{n}$. The nondimensional form of equation (4) becomes

$$
\tilde{f}_{n}=\frac{f_{n}}{N_{0}}=\left(-\tilde{K}_{n} \tilde{x}+\tilde{K}_{3} \tilde{x}^{3}\right) \frac{\left(1+\operatorname{sign}\left(\tilde{x}_{f}-|\tilde{x}|\right)\right)}{2},
$$

with $\tilde{K}_{n}=\frac{K_{n}}{K_{i}}, \tilde{K}_{3}=\frac{K_{3} x_{0}^{2}}{K_{i}}, \tilde{x}_{f}=\frac{x_{f}}{x_{0}}$. Finally, also Eqs. (5), (6) and (7) are rendered nondimensional as follows:

$$
\begin{gathered}
\dot{\tilde{f}}_{s}=(1-\tilde{s}) \tilde{K}_{s}\left[\dot{\tilde{x}}-|\dot{\tilde{x}}| \operatorname{sign}\left(\tilde{f}_{s}-\tilde{\beta}_{s}\right)\left(\frac{\left|\tilde{f}_{s}-\tilde{\beta}_{s}\right|}{\tilde{Y}}\right)^{n_{s}}\right]+\tilde{s} \tilde{K}_{m} \dot{\tilde{x}} \\
\tilde{\beta}_{s}=\tilde{K}_{s} \alpha_{s}\left[\tilde{x}-\frac{\tilde{f}_{s}}{\tilde{\tilde{K}}_{s}}+\tilde{f}_{t} \tanh \left(\tilde{a}_{s} \tilde{x}\right)\left[\frac{1+\operatorname{sign}(-\tilde{x} \tilde{x})}{2}\right]\right] \\
\tilde{s}=\frac{\tanh \left[\tilde{c}_{s}\left(|\tilde{x}|-\tilde{x}_{m}\right)\right]+1}{2}
\end{gathered}
$$


after introducing the following nondimensional parameters:

$\tilde{K}_{s}=\frac{K_{s}}{K_{i}}, \tilde{K}_{m}=\frac{K_{m}}{K_{i}}, \tilde{Y}=\frac{Y}{N_{0}}, \tilde{f}_{t}=\frac{f_{t}}{x_{0}}, \tilde{a}_{s}=a_{s} x_{0}, \tilde{c}_{s}=a_{s} x_{0}, \tilde{x}_{m}=\frac{x_{m}}{x_{0}}$.

\section{Equivalent stiffness and damping}

A useful way to characterize the dynamic behavior of the nonlinear hysteretic oscillator is to make use of the equivalent linearization. An equivalent linear stiffness and an equivalent damping ratio are defined for each displacement amplitude $(U)$ as:

$$
K_{e q}=\frac{\int_{-U}^{U} K_{t} d x}{2 U}, \xi=\frac{E_{d}}{4 \pi E_{k}}
$$

where $K_{t}$ denotes the tangent stiffness at the current displacement $x, E_{d}$ denotes the total dissipated energy and $E_{k}$ is the stored energy for a displacement amplitude $U$. To start with, we analyse the effects of adding SMA wires alone in parallel to the elastomeric isolation system. In order to clarify the effects of the parameters, four different cases for the rheological system $S_{2}$ are reported in Fig. 8: $\left(Y=Z_{m}, \tilde{K}_{s}=\alpha, y_{s}=0.2\right)$ (red dotted line), $\left(Y=Z_{m}, \tilde{K}_{s}=\alpha, y_{s}=0.5\right)($ red dashed line $),\left(Y=Z_{m}, \tilde{K}_{s}=\alpha, y_{s}=0.8\right)($ red solid line), $\left(Y=1.6 Z_{m}, \tilde{K}_{s}=1.6 \alpha, y_{s}=0.8\right)($ red dashed dotted line $)$.
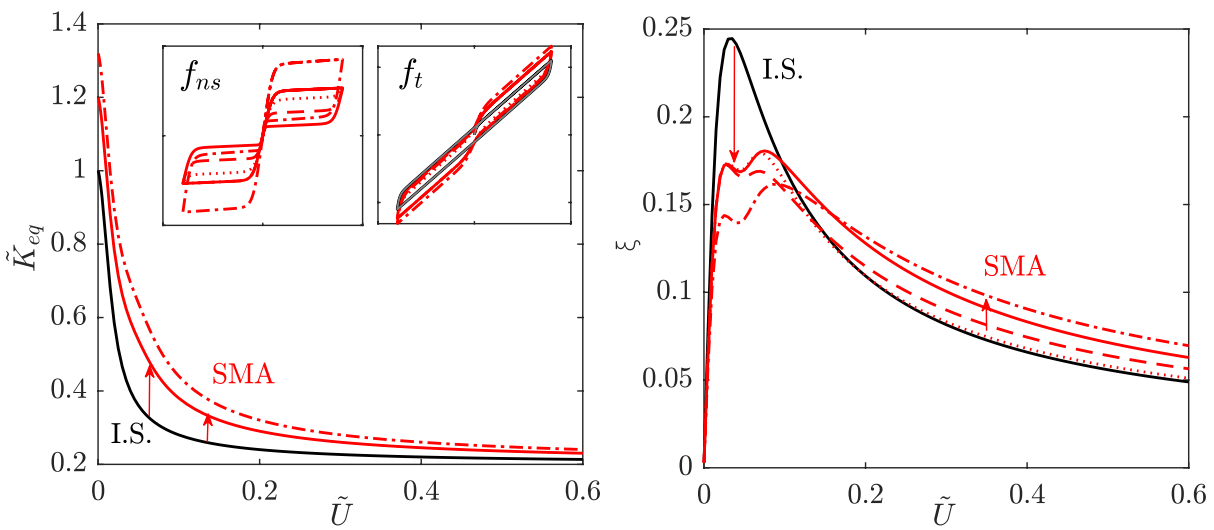

Fig. 8 Equivalent nondimensional stiffness vs. nondimensional displacement amplitude (left) and equivalent damping vs. nondimensional amplitude (right) for $S_{1}$ (black line), $S_{2}$ with $Y=Z_{m}, y_{s}=(0.2,0.5,0.8)$ (dotted, dashed and solid red line, respectively) and with $Y=1.6 Z_{m}, y_{s}=0.8$ (dashed-dotted red line). The sub-figures show the hysteresis loops of device (a) and system (b) for the parameters described above.

The trends of equivalent stiffness and damping in Fig. 8 show that for low ratios of superelastic hysteresis (see dotted red curve) the only effect is an increase of stiffness that causes a reduction of equivalent damping in the low amplitude range. For greater ratios of superelastic hysteresis $\left(y_{s}\right)$, a small increase of damping is achieved for moderate and large displacements, whereas, for low amplitudes, a decrease of damping persists. Increasing the yielding force $(Y)$ entails an increase of initial stiffness and hence damping reduction at low amplitudes is more significant. Moreover, a damping increment for large displacements is affected. Therefore, the introduction of SMA dampers within the isolation system, like any other hysteretic damper, regardless of the rate of hysteresis, causes a decrease in seismic 
isolation performance for low intensity earthquakes but with high frequency return (because of an increase of stiffness and a subsequent decrease of damping), and a slight improvement of performance for medium and high intensity earthquakes. By considering next the case $\left(Y=Z_{m}, y_{s}=0.8\right)$, Fig. 9 shows that the introduction of negative stiffness drastically amplifies the equivalent damping. In fact, while the maximum increase of damping achieved with SMA wires alone was of $\simeq 2 \%$, with $\tilde{K}_{n}=(0.5,1,1.2) \alpha$, the achieved increments are $\simeq(5,25,40) \%$, respectively. As already seen for the case with only SMA wires, also with negative stiffness the increase of hysteretic ratio, $y_{s}$, entails an increase of equivalent damping, while the increase of yielding force $Y$ gives rise to an increase of initial stiffness and thus a drop of equivalent damping over a broad range of displacements.
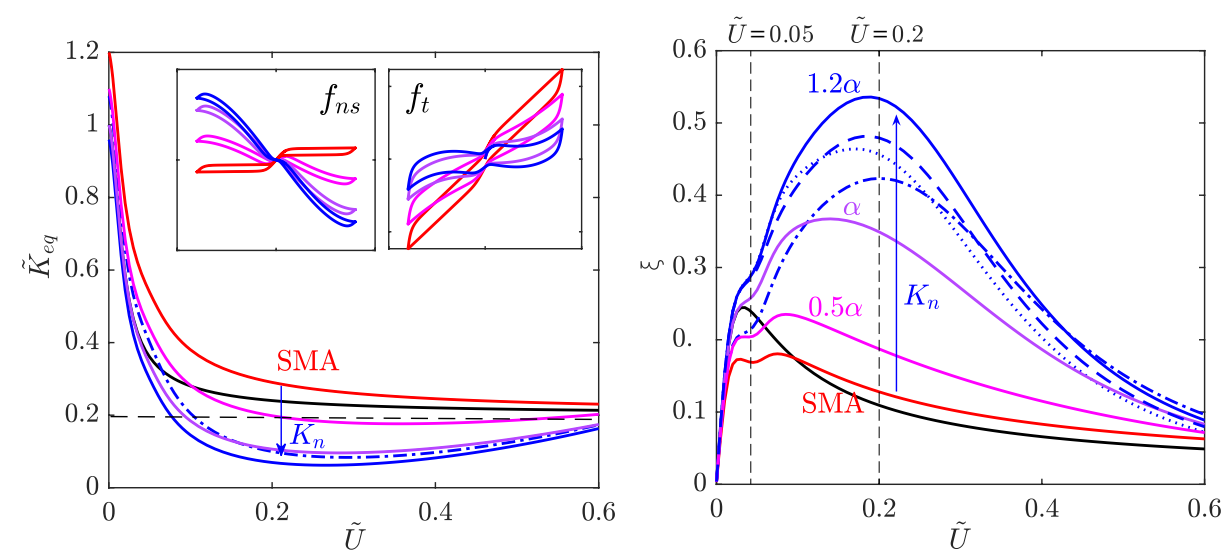

Fig. 9 Equivalent nondimensional stiffness vs. nondimensional displacement amplitude (left) and equivalent damping vs. nondimensional amplitude (right) for $S_{1}$ (black line), $S_{2}$ with $Y=Z_{m}, y_{s}=0.8$ (red line), $S_{3}$ with $\tilde{K}_{n}=(0.5,1,1.2) \alpha$ (magenta, violet, blue lines, respectively); the blue dashed-dotted lines represents the case $\tilde{K}_{n}=1.2 \alpha, Y=1.6 Z_{m}, y_{s}=0.8$, while the subfigures show the hysteresis loops for the assigned parameters.
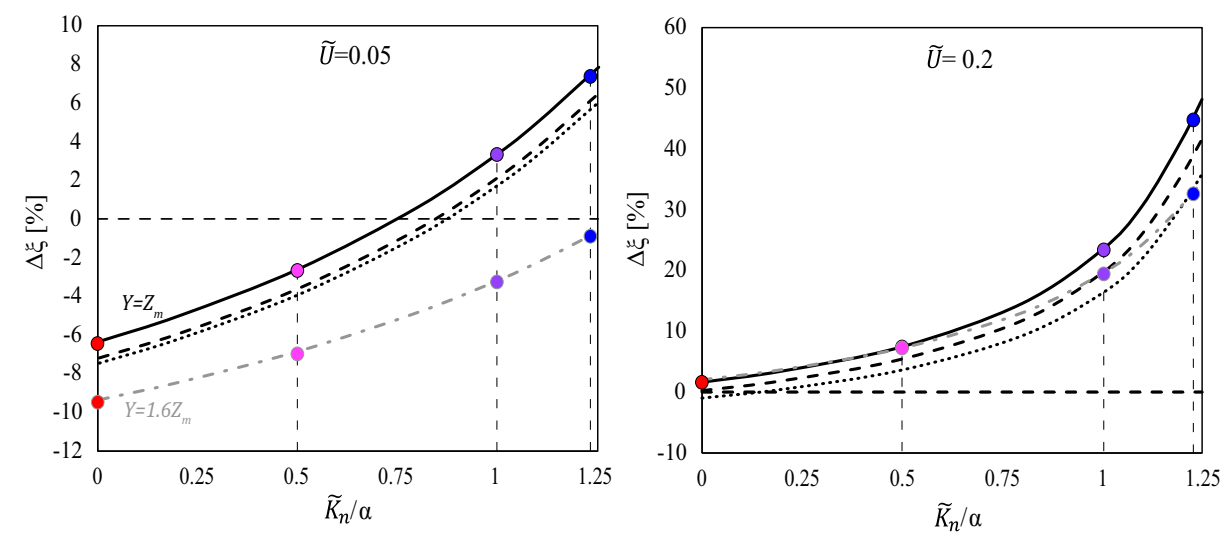

Fig. 10 Damping amplification $\Delta \xi$ vs. negative stiffness coefficient for a nondimensional displacement of 0.05 (left) and of 0.2 (right) for the $S_{3}$ system with $Y=Z_{m}, y_{s}=(0.2,0.5,0.8)$ (black dotted, dashed and solid lines, respectively) and with $Y=1.6 Z_{m}, y_{s}=0.8$ represented by dashed-dotted gray lines. 
In Fig. 10 the damping amplification due to negative stiffness is shown for two different displacement levels equal to $(0.05,0.2) X_{0}$. For the system with the SMA damper without negative stiffness correction, a decrease of damping is observed for low amplitudes while for the second amplitude a slight increase is observed. With the introduction of negative stiffness, a strong damping amplification is achieved for both amplitudes.

By numerically integrating the median line of the hysteresis cycles, the potential energy profile is obtained for the NS-SMA device alone and for the combined system $S_{3}$ (see Fig. 11). The potential energy profile of the device shows its bi-stable/tri-stable nature. In fact, for $\tilde{K}_{n}=(0.2,0.5) \alpha$, the profile has three minima giving rise to three stable equilibria and two unstable equilibria. On the other hand, when the negative stiffness is equal or greater than the SMA elastic stiffness, $\tilde{K}_{n}=(1,1.2) \alpha$, the two unstable equilibria coalesce into one unstable equilibrium point at the origin instead of the original stable equilibrium point and the device becomes bi-stable. The addition of the damper to the isolation system gives rise to an erosion of the global potential energy profile bringing it closer to the damped energy profile, thereby increasing the ratio $E_{d} / E_{k}$, hence, the equivalent damping.
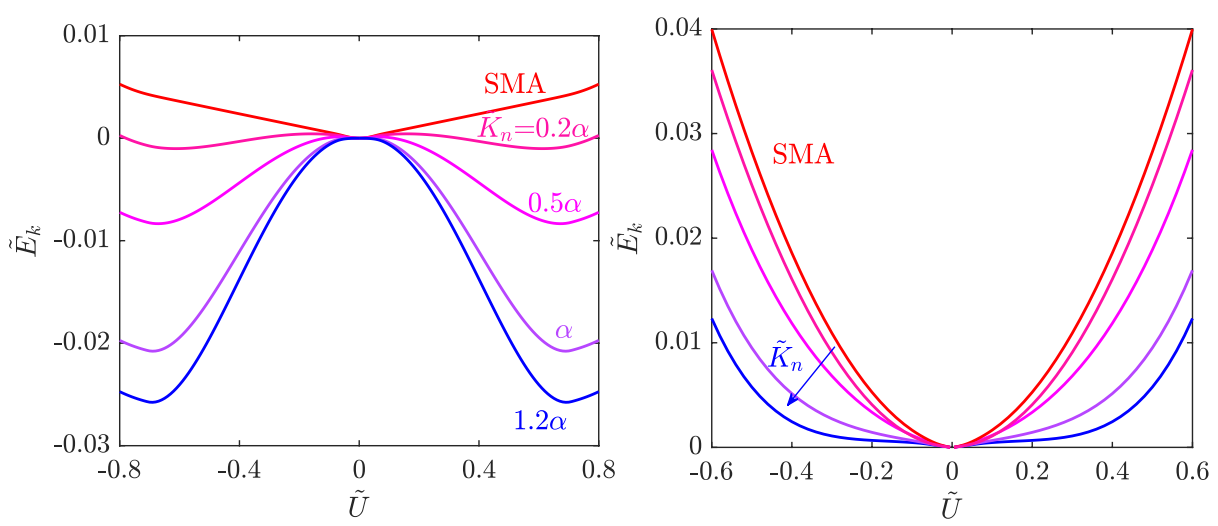

Fig. 11 Potential energy profiles of the NS-SMA damper (left) and of the combined system $S_{3}$ (right) for $\tilde{K}_{n}=(0,0.2,0.5,1,1.2) \alpha$, denoted by red, fuchsia, magenta, violet and blue lines, respectively.

In Fig. 12, it can be seen that for $\tilde{K}_{n}=1.2 \alpha$ the response shows a negative stiffness range and the damped energy profile, along the associated branch, is greater than the potential profile, thus the system becomes overdamped. For values of $\tilde{K}_{n}>1.2 \alpha$, the median line of hysteresis loops intersects the abscissa line (i.e., the force vanishes). Consequently, 2 lateral minima in the energy profile are born, hence the system becomes globally tri-stable. In order to obtain a self-recentering system, the existence of 2 additional equilibrium positions is undesirable. This is why the negative stiffness $\tilde{K}_{n}$ needs to be limited. In the light of the above some useful hints can be obtained on the optimum negative stiffness; indeed, a negative stiffness between $\alpha<\tilde{K}_{n}<1.2 \alpha$ shows a consistent reduction of stiffness and amplification of damping without loss of self-recentering capacity. The limit value of negative stiffness past which the median line of the hysteresis loops intersects the abscissa axis and the system becomes tri-stable can be analytically obtained as

$$
\tilde{K}_{n M S \rightarrow T S}=-\alpha-\alpha_{s} \tilde{K}_{s}-3 \sqrt[3]{\frac{\left(-1+\alpha_{s}\right)^{2} \tilde{K}_{3} \tilde{K}_{s}^{2} \tilde{x}_{g}^{2}}{4}}
$$




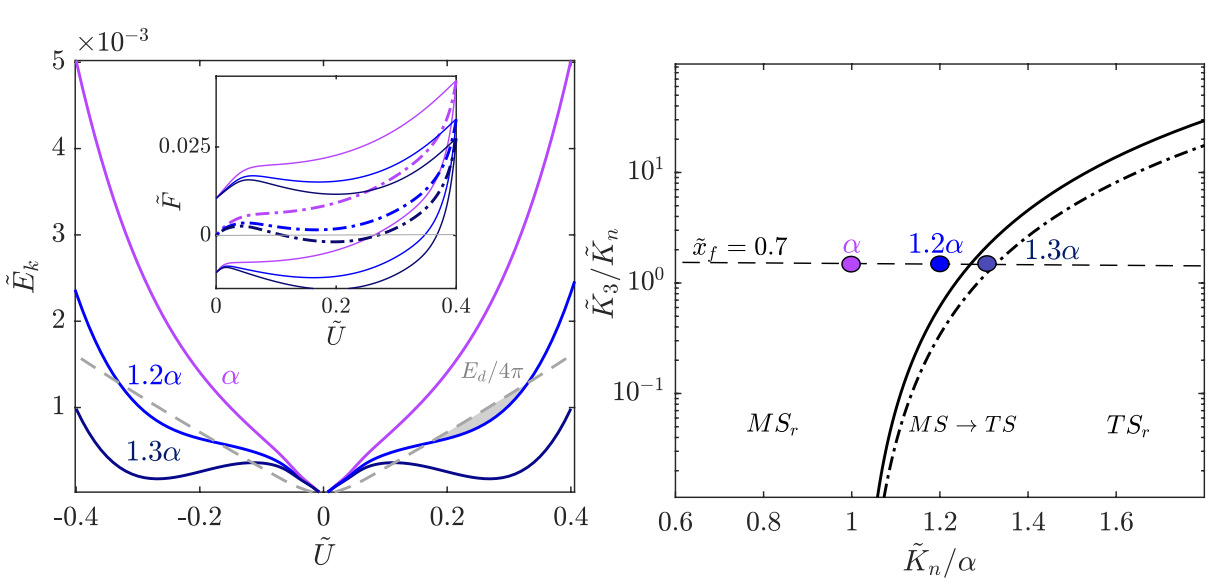

Fig. 12 (left) Potential energy profiles of system $S_{3}$ with $\tilde{K}_{n}=(1,1.2,1.3) \alpha$ represented by magenta, blue, and violet lines, respectively and damped energy profile denoted by gray dashed lines. The subfigure shows the total force displacement cycles together with the average force in dashed-dotted lines. (right) Analytical bounding curves between mono-stability and tri-stability in the parameters space of the negative stiffness mechanism $\left(\tilde{K}_{n}, \tilde{K}_{3}\right)$ for the $S_{3}$ system with $y_{s}=0.8$ and $Y=(1,1.6) Z_{m}$ represented by black solid and black dashed-dotted lines, respectively.

\section{Nonlinear dynamic response scenario}

The FRCs of the described nonlinear hysteretic oscillators endowed with the rheological devices $S_{1}, S_{2}, S_{3}$ are numerically obtained for several excitation levels employing a continuation procedure based on the Poincarè map. The Poincarè map and the associated monodromy matrix are computed via the fourth order Runge-Kutta integration scheme and the finite difference method, respectively. Monitoring the eigenvalues of the monodromy matrix (i.e., Floquet multipliers) allows to ascertain the stability and bifurcations along the path of periodic solutions $[38,39]$. In the next subsections, a full parametric analysis is carried out to investigate the sensitivity of the frequency-response with respect to the main design parameters.

Linear and nonlinear negative stiffness. In Fig. 13, the displacement and acceleration FRCs are reported for the baseline isolation system $\left(S_{1}\right.$ system denoted by black lines), for the isolation system with the SMA damper $\left(S_{2}\right.$ system with $\tilde{K}_{s}=\alpha, \tilde{x}_{g}=0.05, y_{s}=0.2$, denoted by red lines) and for the isolation system with the same SMA damper plus the negative stiffness $\left(S_{3}\right.$ system with $\left.\tilde{K}_{n}=(0.5,1,1.2) \alpha\right)$ for two different excitation amplitudes, $\tilde{A}_{g}=(0.01,0.015)$. As expected, the addition of SMA wires induces an increase of stiffness, larger at low amplitudes, resulting in a shift of the curves to the right, and then an increase of accelerations and a decrease of displacements. With the introduction of negative stiffness, we observe a reverse shift of the curves to the left and then a decrease of accelerations and an increase of displacements. It is interesting to note that despite the stiffness being much lower than the baseline system, the maximum displacement is always smaller, at most equal, to that of the original system, thanks to the beneficial effect of the augmented damping. Besides the negative stiffness coefficient, also the nonlinear stiffness coefficient $\tilde{K}_{3}$ plays an important role on the nonlinear dynamic response. In Fig. 14, the displacement and acceleration FRCs are reported for the baseline isolation system (black line) and for the $S_{3}$ system endowed with different nonlinear stiffness coefficients. 

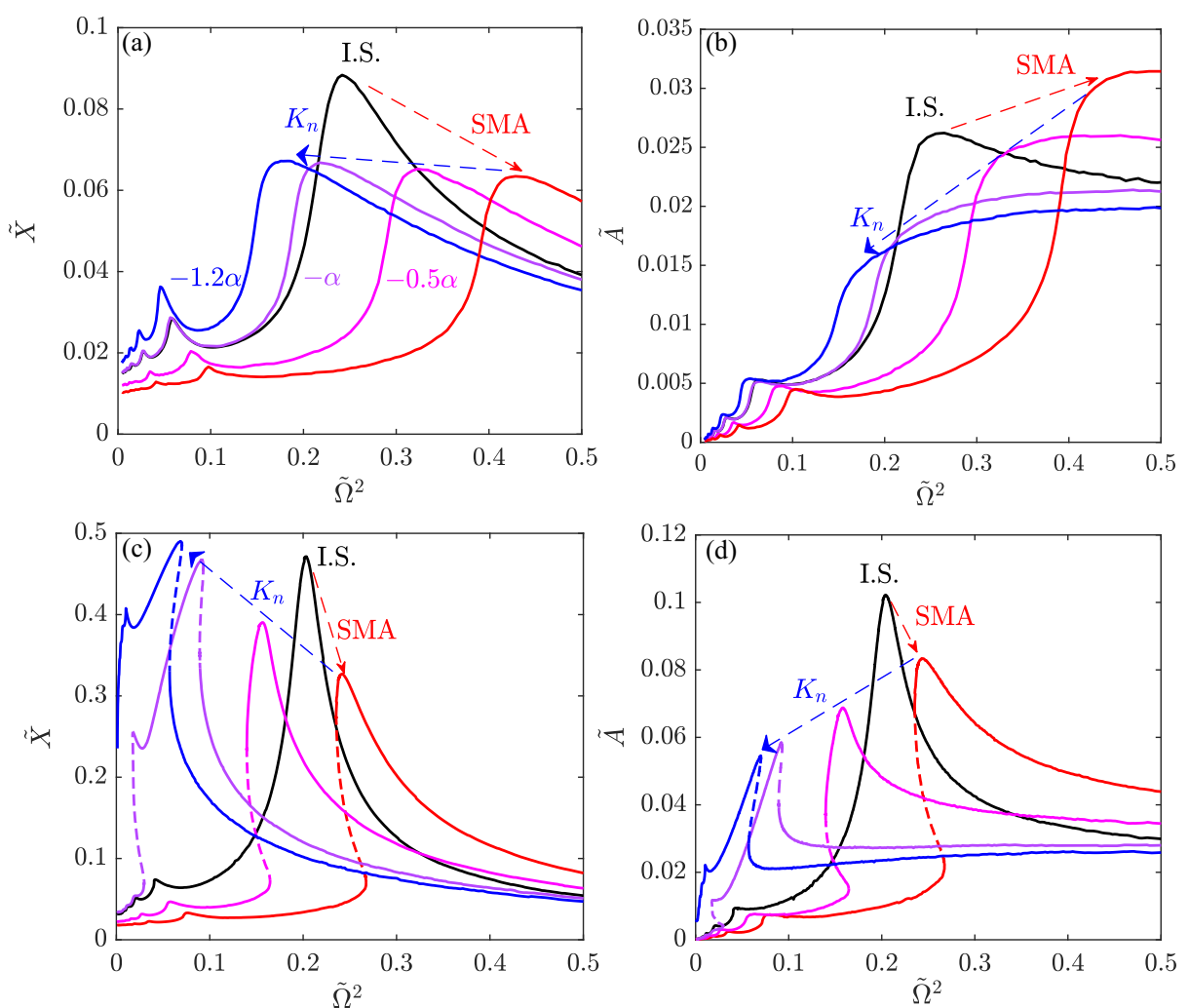

Fig. 13 Frequency-response curves (FRCs) in terms of nondimensional displacement (left) and acceleration (right) for a ground acceleration of 0.01 ((a) and (b)) and 0.015 ((c) and (d)). The response of $S_{1}$ is denoted by black lines, the response of $S_{2}$ (when $Y=Z_{m}, y_{s}=0.2$ ) by red lines while the response of $S_{3}$ by magenta $\left(\tilde{K}_{n}=0.5 \alpha\right)$, violet $\left(\tilde{K}_{n}=\alpha\right)$ and blue lines $\left(\tilde{K}_{n}=1.2 \alpha\right)$, respectively.
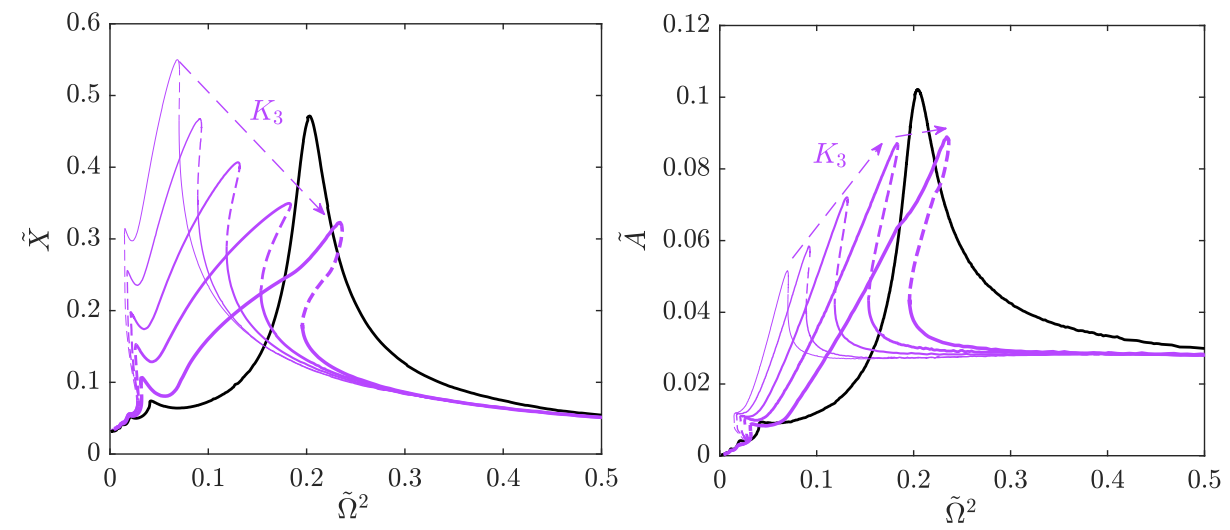

Fig. 14 Frequency-response curves (FRCs) in terms of nondimensional displacement (left) and acceleration (right) for a ground acceleration set to 0.015 . The response of $S_{1}$ is represented by black lines and that of $S_{3}$ (when $Y=Z_{m}, y_{s}=0.2, \tilde{K}_{n}=\alpha$ and $\left.\tilde{K}_{3}=(1,2,4,8,10) 10^{-6} \tilde{K}_{n}\right)$ are denoted by solid violet lines with increasing thickness for increasing $\tilde{K}_{3}$. 
Note that an increase of $\tilde{K}_{3}$, associated with a smaller working displacement $\tilde{x}_{f}$, entails a stronger hardening nonlinearity that leads to a reduction of the peak displacement and to an increase of the peak acceleration.

SMA mechanical characteristics. In Fig. 15 the FRCs of the baseline isolation system (black lines) are compared with those of the $S_{2}$ system (red lines) and with those of the $S_{3}$ system (violet lines) for different levels of hysteresis ratio $y_{s}$ and for two excitation amplitudes, $\tilde{A}_{g}=(0.01,0.015)$. The acceleration of the $S_{2}$-isolated system shows, for low excitations and regardless of the hysteresis ratio, an increase of acceleration compared to the baseline $S_{1}$ system. On the contrary, the $S_{3}$-isolated system exhibits for both excitation amplitudes a strong reduction in accelerations while for medium and high hysteresis ratios of the SMA damper, it also undergoes a strong reduction in displacements. By introducing more SMA wires (with $Y=1.6 Z_{m}$ ), an additional reduction of displacement amplitude can be achieved but at the expense of a stiffness increase and, accordingly, of accelerations transmissibility.
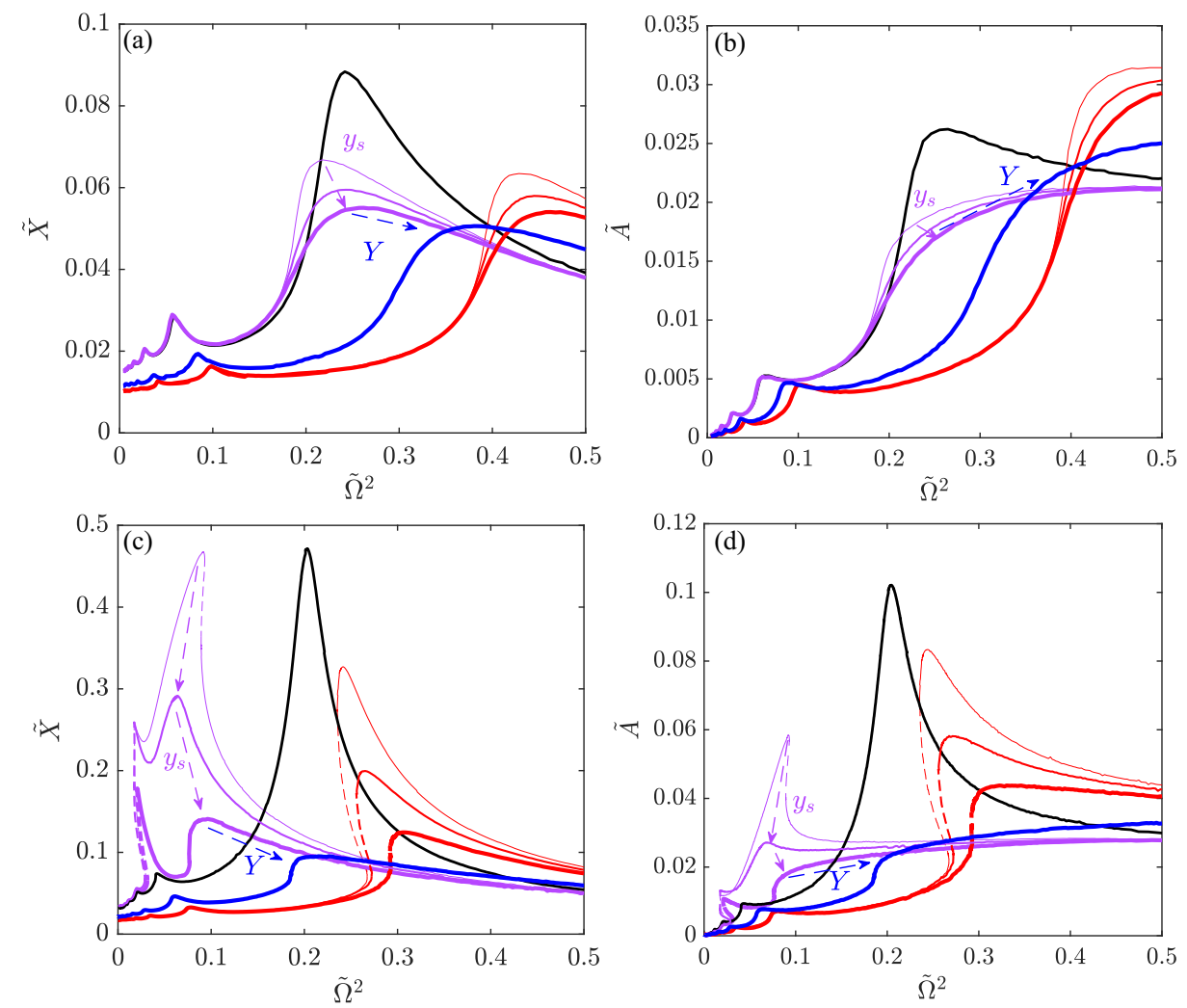

Fig. 15 Frequency-response curves in terms of nondimensional displacement (left) and acceleration (right) for a ground acceleration set to $0.01\left((\mathrm{a})\right.$ and (b)) and $0.015\left((\mathrm{c})\right.$ and (d)). The response of the $S_{1}$-isolated system is described by black lines, those of $S_{2}$ (when $Y=Z_{m}, y_{s}=(0.2,0.5,0.8)$ ) by red lines with increasing thickness for increasing $y_{s}$, and those of $S_{3}$ (when $\tilde{K}_{n}=\alpha, Y=Z_{m}, y_{s}=(0.2,0.5,0.8)$ ) by violet lines with increasing thickness for increasing $y_{s}$ and blue lines $\left(\tilde{K}_{n}=\alpha, Y=1.6 Z_{m}, y_{s}=0.8\right)$, respectively. 


\section{Displacement and acceleration transmissibilities}

The parametric study has disclosed a meaningful sensitivity of the frequency-response with respect to the system parameters. Henceforth, the evolution of the response for increasing base accelerations is discussed. The FRCs of the $S_{3}$-isolated system, are computed for different excitation amplitudes (see Fig. 16). The strong softening-hardening nonlinearity of the system is reflected by the trend of the system backbone curves. Moreover, other interesting phenomena are observed such as the emergence of detached resonance curves. For the case with large negative stiffness $\left(\tilde{K}_{n}=1.2 \alpha\right)$ there occurs a disappearance of the peak in the response within the displacement range in which the system turns out to be overdamped $(0.2<\tilde{x}<0.4)$. Moreover, for the strongest base acceleration in the low frequency range, there exists a bandwidth in which no stationary solutions could be obtained, a circumstance that suggested the existence of quasi-periodic/nonperiodic responses.
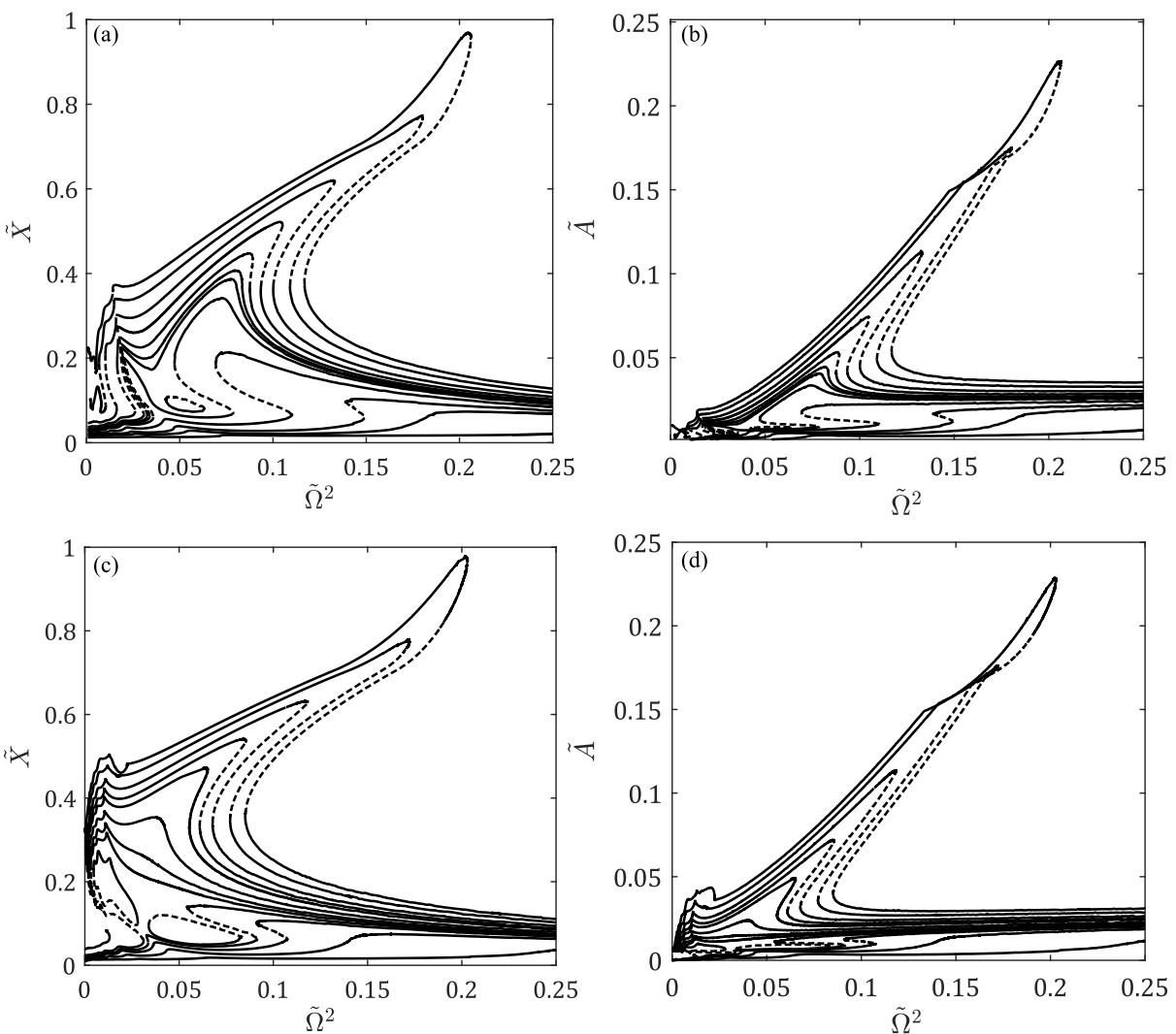

Fig. 16 FRCs in terms of nondimensional displacement (left) and acceleration (right) for the $S_{3}$ system with $\tilde{K}_{n}=\alpha, Y=Z_{m}, y_{s}=0.2$ (top) and with $\tilde{K}_{n}=1.2 \alpha, Y=Z_{m}, y_{s}=0.2$ (bottom) when the base accelerations are set to $(0.8,1,1.08,1.2,1.28,1.3,1.32,1.36,1.4,1.48,1.6,1.8,2) 10^{-2}$.

For the same excitation amplitudes, the FRCs are computed for the baseline isolation system, for the $S_{2}$-isolated system with various hysteresis ratios and yielding force levels, and for the $S_{3}$-isolated system with various negative stiffness and hysteresis levels. The ratios 
between the peak response of the $S_{2}$ or $S_{3}$-isolated system and the response of the baseline system are reported in Fig. 17.
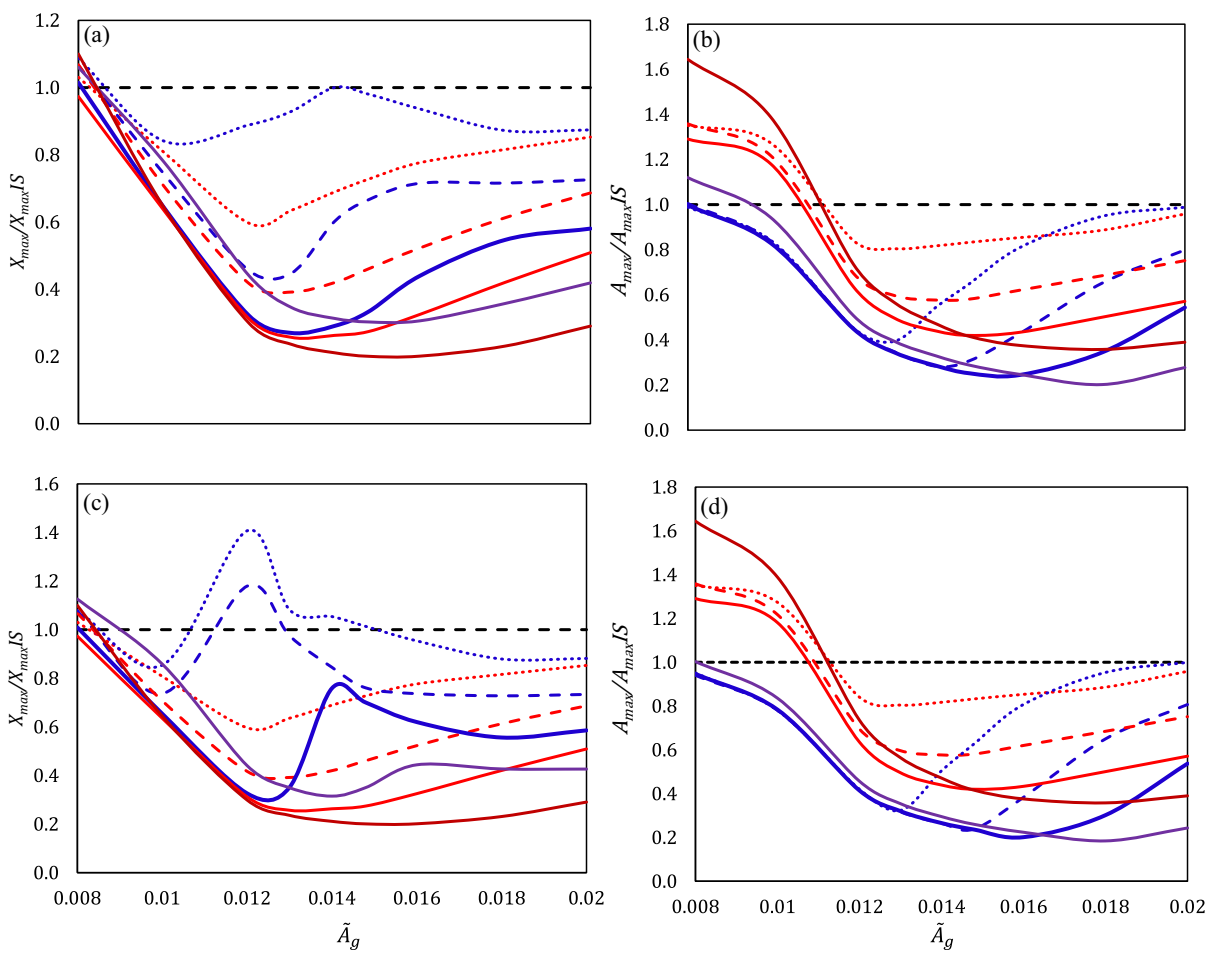

Fig. 17 Ratio between the peak response of the controlled system and that of the baseline system in terms of nondimensional displacements (left) and accelerations (right). The responses of $S_{2}$ with $Y=Z_{m}$ and $y_{s}=$ $(0.2,0.5,0.8)$ are represented by red dotted, red dashed and red solid lines, respectively, while the response of $S_{2}$ with $Y=1.6 Z_{m}$ and $y_{s}=0.8$ is described by bordeaux solid lines. The responses of $S_{3}$ with $\tilde{K}_{n}=\alpha$ (top), $1.2 \alpha$ (bottom), $Y=Z_{m}, y_{s}=(0.2,0.5,0.8)$ are described by blue dotted, blue dashed and blue solid lines, respectively, while the response of $S_{3}$ with $\tilde{K}_{n}=\alpha$ (top), $1.2 \alpha$ (bottom), $Y=1.6 Z_{m}, y_{s}=0.8$ is indicated by violet solid lines.

As expected, the insertion of the SMA damper alone causes an increase of accelerations response for weak excitations. The increase is about $30 \%$ when $Y=Z_{m}$ and $60 \%$ when $Y=1.6 Z_{m}$, respectively. Hence, it depends mainly on the initial stiffness of the hysteretic damping and mildly on the damping ratio. On the other hand, the damping ratio strongly affects the response for moderate to strong base accelerations. It turns out that reductions of $20 \%, 40 \%, 60 \%$ are obtained when $y_{s}=(0.2,0.5,0.8)$, respectively. By introducing the negative stiffness in parallel with the SMA damper in the $S_{3}$ system, the amplification of accelerations for weak excitations is totally cancelled and a mild reduction can be observed. In addition, a further $20 \%$ reduction of accelerations compared with the $S_{2}$-isolated system is obtained for moderate and strong base excitations, achieving an overall acceleration reduction of $40 \%, 60 \%$, and $80 \%$ for $y_{s}=(0.2,0.5,0.8)$, respectively. The trend in acceleration reduction coincides with the trend depicting stiffness reduction. Therefore, it shows a bell shape with a peak corresponding to the displacement where the stiffness reduction is maximum. Given the presence of a cubic stiffness in the NS mechanism, for large displace- 
ments, the equivalent stiffness, hence the maximum acceleration, reaches again the stiffness of the baseline system. The effect of increasing the damping ratio is an expansion of the bell, both in terms of width and height. If the initial stiffness of the SMA damper overcomes the negative stiffness, as is the case with $\tilde{K}_{n}=\alpha$ and $Y=1.6 Z_{m}$ (violet line in Fig. 17b), a slight increase of peak acceleration for low excitations occurs, together with an expansion of the bell width and of the effective displacement range. By balancing the increase of SMA damper's initial stiffness with an equivalent increase of negative stiffness as is the case with $\tilde{K}_{n}=1.2 \alpha$ and $Y=1.6 Z_{m}$, (violet line in Fig. 17d), the increase of peak acceleration for low excitation amplitudes is cancelled again. For moderate and strong excitations, the increase of negative stiffness from $\alpha$ to $1.2 \alpha$ causes a slight increase of acceleration reduction of about $10 \%$. Regarding the displacements, it is worth highlighting a substantial coincidence between the reduction offered by the SMA system and by the NS-SMA system with $\tilde{K}_{n}=\alpha$ for weak base excitations. The trend in reduction is for both systems initially quasilinear up to a maximum value corresponding to the displacement that yields the maximum damping. The maximum displacement reduction is quite similar for both systems except for low damping ratios $\left(y_{s}=0.2\right)$ where the stiffness reduction is not balanced by a robust damping augmentation and it is of about $20 \%$ for the $S_{3}$ system and of about $40 \%$ for the $S_{2}$ system. For the remaining damping ratios, the displacement peak reduction for both systems is about $55 \%$ and $65 \%$ with $y_{s}=(0.2,0.5)$, respectively. Past the maximum, the trend of peak displacement reduction of the $S_{3}$-isolated system deviates from the trend of the $S_{2}$ system, showing a smaller reduction. The maximum deviation between the two responses occurs where the stiffness reduction is maximum.
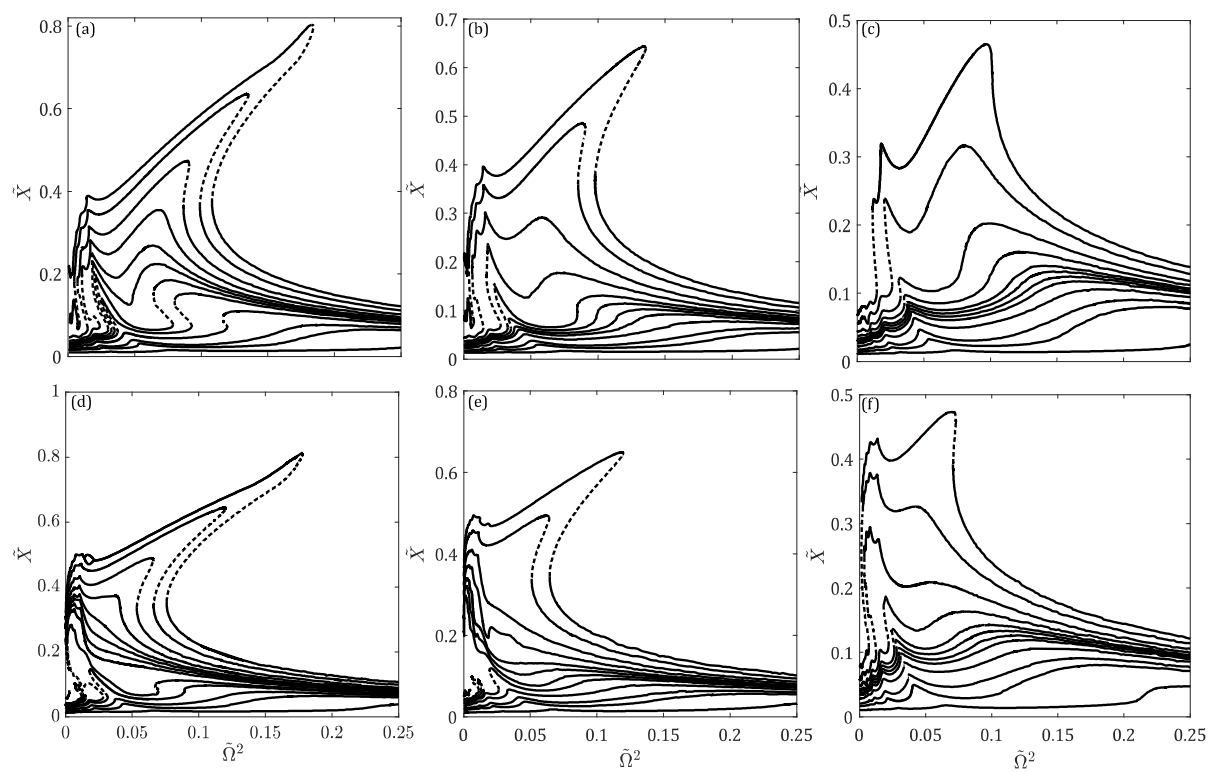

Fig. $18 \mathrm{FRCs}$ in terms of nondimensional displacements for the base accelerations set to $(0.8,1,1.08,1.2,1.28,1.3,1.32,1.36,1.4,1.48,1.6,1.8,2) 10^{-2}$ for the $S_{3}$ system with $\tilde{K}_{n}=\alpha, Y=Z_{m}, y_{s}=$ $(0.5,0.8)$ (parts (a) and (b)) and $\tilde{K}_{n}=\alpha, Y=1.6 Z_{m}, y_{s}=0.8$ (part (c)), and with $\tilde{K}_{n}=1.2 \alpha, Y=Z_{m}, y_{s}=$ $(0.5,0.8)$ (parts (d) and (e)) and $\tilde{K}_{n}=\alpha, Y=1.6 Z_{m}, y_{s}=0.8$ (part (f)). 
The increase of yielding force $(Y)$, hence of the initial SMA stiffness, from $Y=Z_{m}$ to $Y=1.6 Z_{m}$ moves the curves to the right, showing a smaller reduction for weak excitations and a larger reduction for moderate and strong base excitations. For the case with $\tilde{K}_{n}=1.2 \alpha$, the response reduction is smaller than that achieved with $\tilde{K}_{n}=\alpha$, while for a certain range of base excitations, there exists an increase in response. This range of base excitations corresponds to the FRCs where the displacement peak is due to the superharmonic resonance, as one can note in Fig. 18.

Next we address the force transmissibility in terms of frequency bandwidth where effective isolation is attained. We consider force transmissibility as the ratio between absolute acceleration and base acceleration. As known, a response is considered effectively controlled when the transmissibility is lower than 1 . For the nondimensional base acceleration of 0.02 , the acceleration peak reduction for the $S_{3}$-isolated system is minimum (i.e., $40 \%$ ) and is equal to that of the $S_{2}$-isolated system (see Fig. 17b and Fig. 17d). By analysing the force transmissibility under the same base accelerations, further useful considerations can be drawn about the isolation performance of the proposed system. While Fig. 17 shows only the reduction in acceleration peak, Fig. 19 shows the bandwidth of the isolated frequencies.

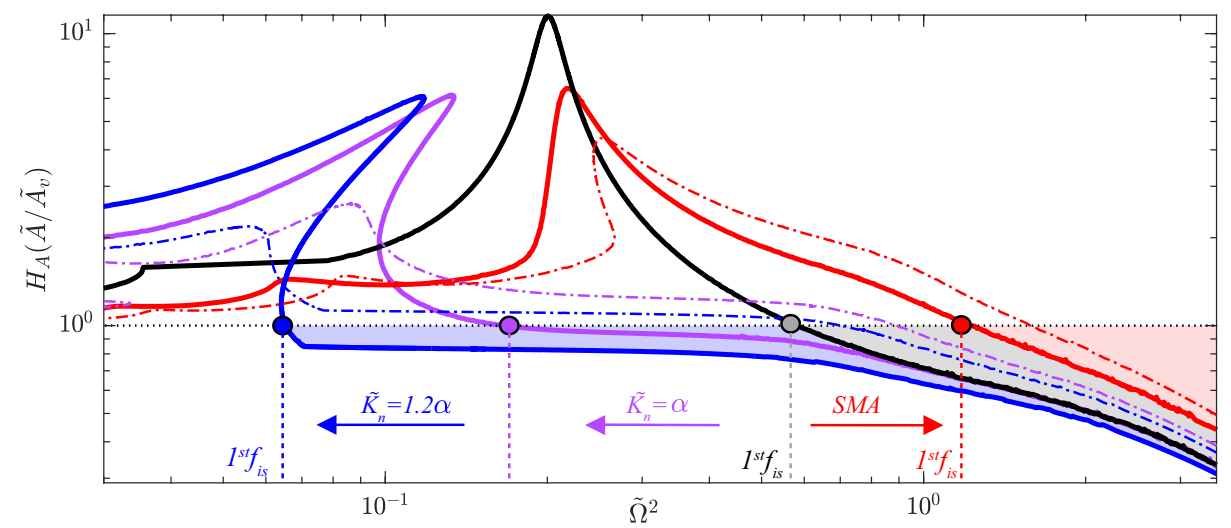

Fig. 19 FRCs in terms of force transmissibility for a nondimensional base acceleration equal to 0.02. The response of the $S_{1}$-isolated system is described by the black solid line, while the responses of the $S_{2}$ system (when $y_{s}=0.8, Y=(1,1.6) Z_{m}$ ) are denoted by the red solid and red dashed lines, respectively. The responses of the $S_{3}$-isolated system (when $\tilde{K}_{n}=\alpha, Y=(1,1.6) Z_{m}$ ) are described by the violet solid and dashed lines and those for the $S_{3}$ system (when $\tilde{K}_{n}=1.2 \alpha, Y=(1,1.6) Z_{m}$ ) are described by the blue solid and blue dashed lines, respectively. In all curves $y_{s}=0.8$.

The introduction of the SMA damper alone increases the value of the first isolated frequency, thus reducing the bandwidth of isolated frequencies of $69 \%$ and $87 \%$ for $Y=Z_{m}$ and $Y=1.6 Z_{m}$, respectively. On the other hand, the negative stiffness mechanism in parallel with the SMA damper, in addition to a reduction of the peak response, yields an increase of bandwidth of isolated frequencies, reducing the value of the first isolated frequency of $25 \%$ and $44 \%$ with $\tilde{K}_{n}=\alpha$ and $\tilde{K}_{n}=1.2 \alpha$, respectively. It is also possible to observe that an increase of the SMA yielding force $(Y)$ entailing an increase of initial SMA stiffness not accompanied by an increase of negative stiffness, yields a further reduction in peak response but at the expense of a reduction of isolated frequency bandwidth. 


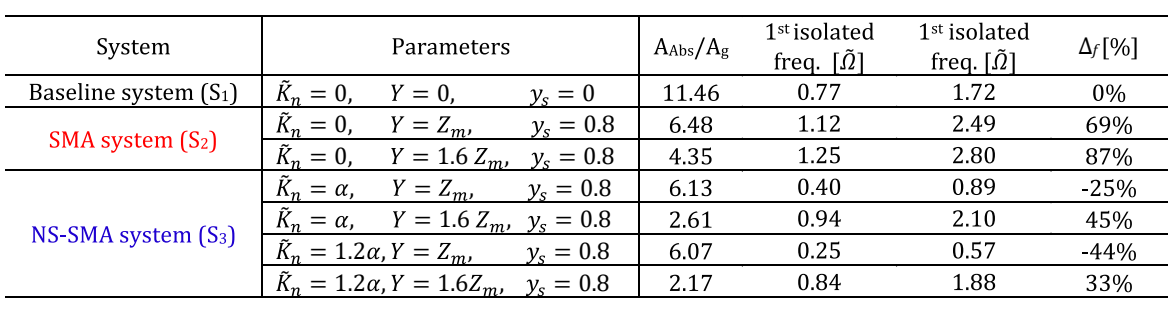

Fig. 20 Force transmissibility performance for the three kinds of isolated systems, $S_{1}, S_{2}$ and $S_{3}$.

\section{Primary, superharmonic and detached resonances}

The severe softening nonlinearity associated with the softening hysteresis induces an interaction between the primary and the superharmonic resonances causing the emergence of detached resonance curves, a phenomenon that is well documented in the literature [40, 41, 42]. However, a new phenomenology is here documented. In Fig. 21 the evolution of the isolas for the system with two levels of negative stiffness is shown. The qualitative pattern in both cases consists in the birth of an outer isola in the neighborhood of the superharmonic resonance frequency which, for increasing base acceleration levels, coalesces first with the superharmonic resonance branch and thereafter, upon further increase of excitation amplitude, with the main resonance branch, giving rise to an inner island.
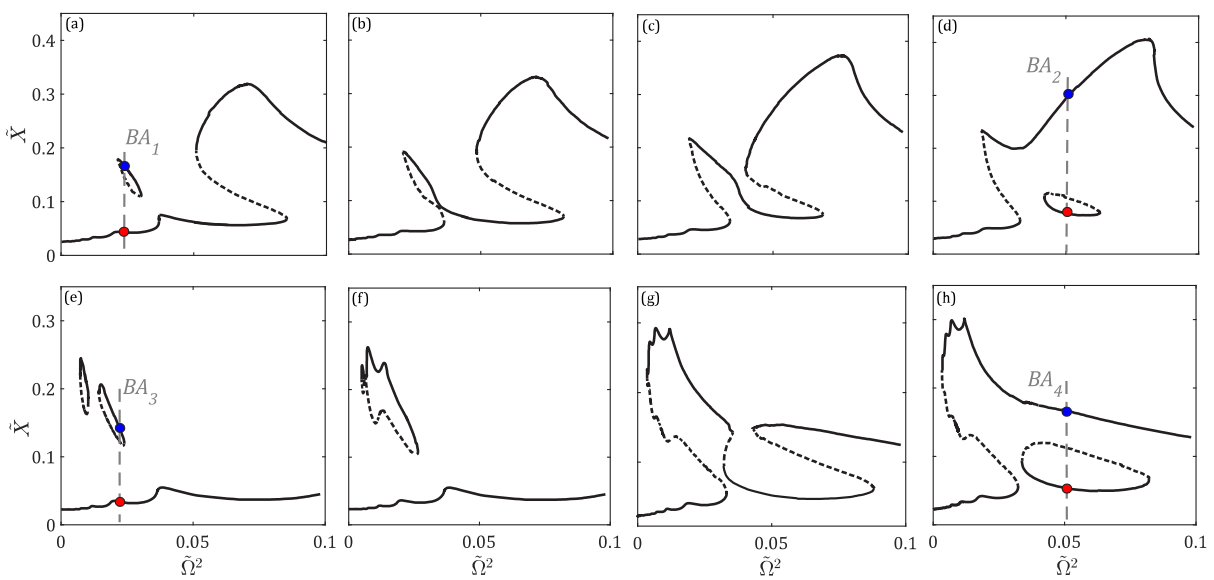

Fig. 21 Evolution of isolas topology for the $S_{3}$ system with $\tilde{K}_{n}=\alpha, Y=Z_{m}, y_{s}=0.2$ (top) for a nondimensional ground acceleration equal to 0.0128 (a), 0.01288 (b), 0.0130 (c), 0.0132 (d) and for the $S_{3}$ system with $\tilde{K}_{n}=1.2 \alpha, Y=Z_{m}, y_{s}=0.2$ (bottom) for a nondimensional ground acceleration equal to 0.01072 (e), 0.01088 (f), $0.01112(\mathrm{~g})$ and $0.0112(\mathrm{~h})$.

It is possible to observe that for the $S_{3}$ system with $\tilde{K}_{n}=\alpha$, there exists only a small outer island, while for the case with $\tilde{K}_{n}=1.2 \alpha$, different outer islands coexist. Indeed, for a ground acceleration of 0.01 , two outer detached resonance isolas are visible in the proximity of the superharmonic resonances of order 1:3 and 1:5 (see Fig. 21e). For a higher amplitude, the two distinct isolas merge and a new isola is formed near the superharmonic resonance of order 1:7 (see Fig. 21f). Upon further increasing the excitation amplitude, all previous isolas 
merge with each other and with the 1:3 superharmonic resonance branch (see Fig. 21g). At a higher excitation amplitude, the primary resonance branch and the outer superisola merges and give rise to an inner isola for slightly higher excitation amplitudes (see Fig. 21h). Despite the very low frequency range where the isolas appear, the outer isolas are detrimental for isolation purposes since they can give rise to unwanted dynamic amplification. On the contrary, the inner islands can be used to reduce the response near the main frequency. The factors that determine whether the mass will move along the detached solution curve or along the main branch are the initial conditions or the perturbations causing jumps between the coexisting attractors.

In order to obtain basins of attraction of the system near the isolas (see Fig. 22), the equations of motion are numerically integrated for a harmonic base excitation over 1,000 periods over a grid of initial conditions. The initial conditions, in terms of displacement and velocity, that lead to different attractors are denoted by different colors. In particular, the initial conditions that lead to the low-amplitude solution are represented in red, while in blue those that lead to the high-amplitude solution. It is possible to note that the system with $\tilde{K}_{n}=\alpha$ shows much thinner basins of attraction for both the outer and inner isola than the system with $\tilde{K}_{n}=1.2 \alpha$, denoted by a major blue portion and a major red portion for the outer and inner isolas, respectively.

(a)

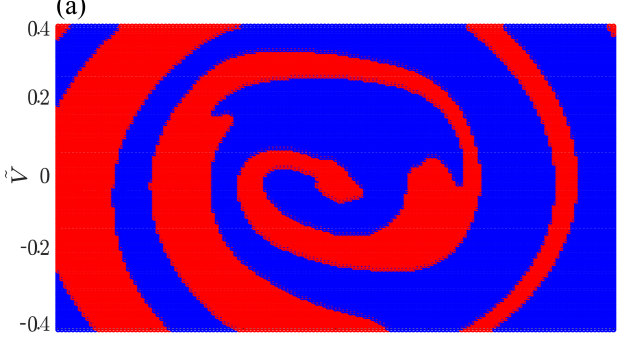

(c)

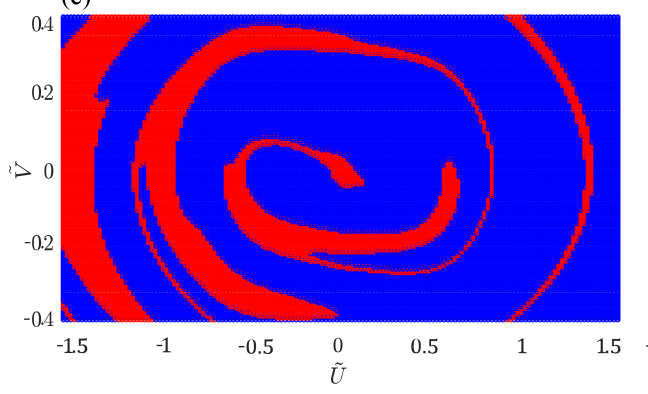

(b)

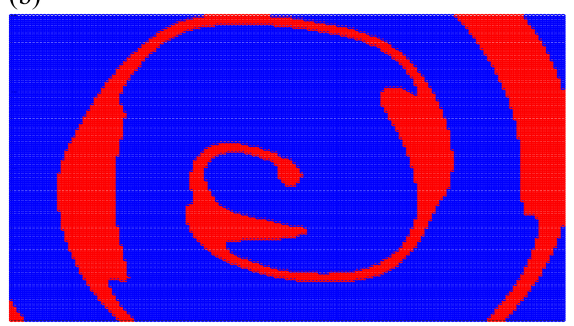

(d)

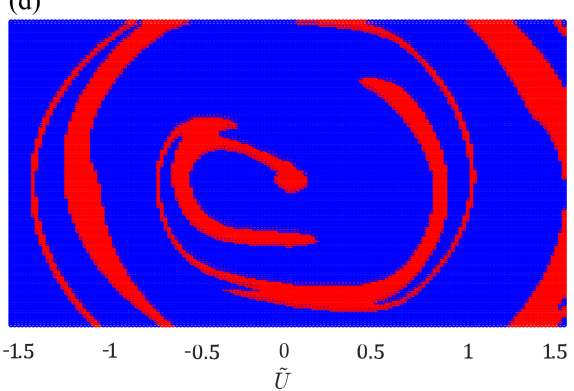

Fig. 22 Basins of attraction for the system with $\tilde{K}_{n}=\alpha, Y=Z_{m}, y_{s}=0.2$ (top) for a base acceleration $\tilde{A}_{g}=0.0128$ and frequency $\tilde{\Omega}^{2}=0.022$, corresponding to the outer isola (left), and for a base acceleration $\tilde{A}_{g}=0.0132$ and frequency $\tilde{\Omega}^{2}=0.05$, corresponding to the inner isola (right). (bottom) Basins of attraction for the system with $\tilde{K}_{n}=1.2 \alpha, Y=Z_{m}, y_{s}=0.2$ for a base acceleration of $\tilde{A}_{g}=0.01072$ and $\tilde{\Omega}^{2}=0.022$, corresponding to the outer isola (left), and for a base acceleration of $\tilde{A}_{g}=0.0112$ and $\tilde{\Omega}^{2}=0.05$, corresponding to the inner isola (right). In red the initial conditions that lead to the low-amplitude solution, while in blue those that lead to the high-amplitude solution.

For a more thorough characterization of the two coexisting attractors, one along the main curve and the other along the detached curve, exhibited by the system with $\tilde{K}_{n}=1.2 \alpha, Y=$ 
$Z_{m}, y_{s}=0.2$, the force-displacement cycles, the phase portraits, the time histories and the FFTs of the response to a harmonic base excitation with $\tilde{A}_{g}=0.01072$ and $\tilde{\Omega}^{2}=0.022$ are shown in Fig. 23. The response belonging to the main solution branch is richer due to the presence of more superharmonic components. In fact, while in the first case, third, fifth and seventh harmonics exist, in the case of the isola only the third harmonic is manifested. From the FFT it is also possible to see that, for solutions belonging to the isola, the amplitude of the main harmonic is larger than that of the response, sum of all harmonics, while the solution along the main curve shows the fundamental harmonic with the same amplitude of the response. This suggests for the two solutions a different relative phase between the main harmonic and higher harmonics. Through a harmonic decomposition of the two different responses shown in Fig. 24, it is possible to see that, for the solution belonging to the main branch, the peak of main harmonic corresponds to the zero of all other harmonics having thus a relative phase of $\pi / 2 n$ with $n$ representing the order of the harmonic component. By focusing on the third harmonic, a relative phase of $\pi / 6$ means that the peak of the main harmonic coincides with the zero value with negative derivative of superharmonic and this is equivalent to the out-of-phase condition or deamplification condition. On the other hand, the solution along the detached curve shows that the peak of the main harmonic coincides with the minimum of the third harmonic, thus giving rise to a $\pi / 2$ relative phase. It can be said that, for the solutions belonging to the detached curve, the higher harmonics are phased with the main harmonic, producing a less distorted and larger motion.
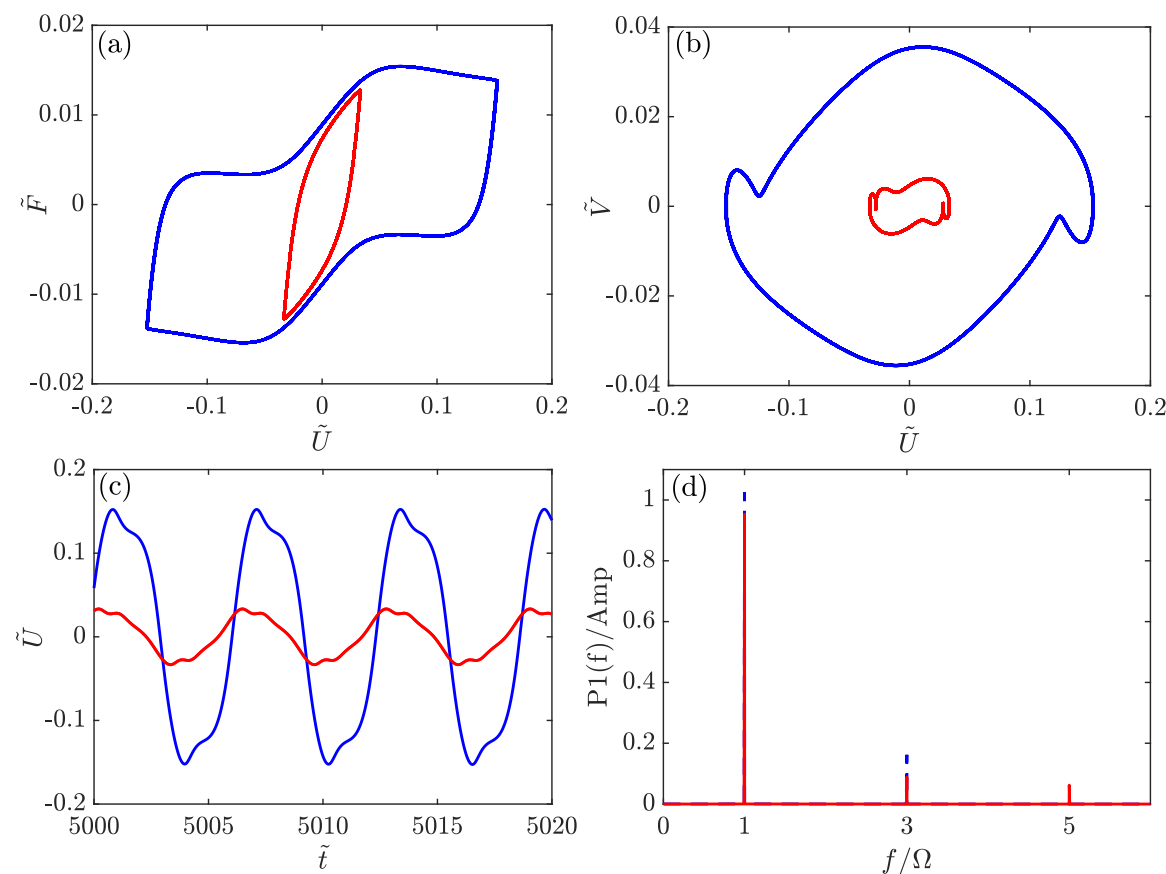

Fig. 23 Force-displacement cycles (a), phase portraits (b), time histories (c) and FFTs (d) of the S3 system (with $\tilde{K}_{n}=1.2 \alpha, Y=Z_{m}, y_{s}=0.2$ ) for $\tilde{A}_{g}=0.01072$ and $\tilde{\Omega}^{2}=0.022$. In red the response to a zero initial condition along to the main solutions branch, while in blue the solution for the initial conditions $\tilde{x}=0.2, \tilde{v}=0$, giving rising to the isola solution curve. 

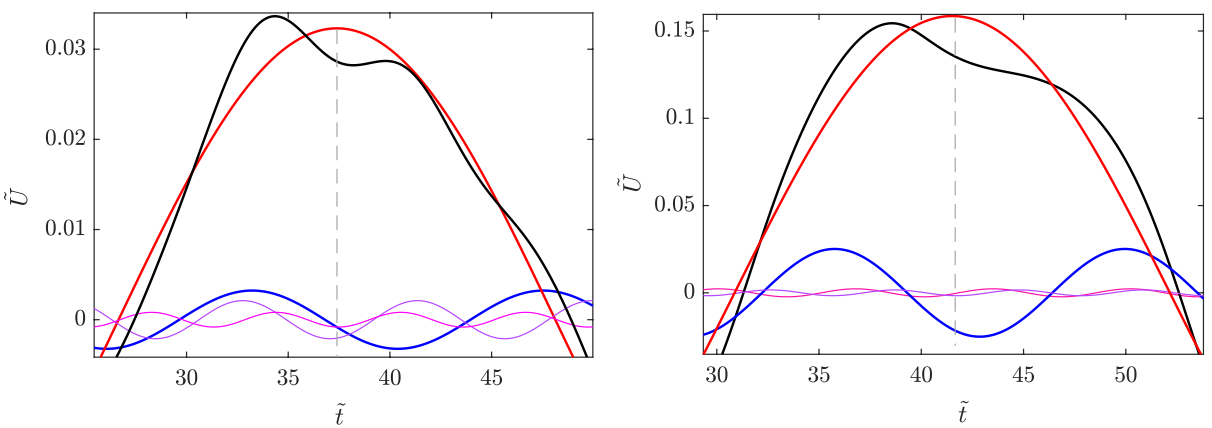

Fig. 24 Harmonic decomposition of the superharmonic response along the main resonance branch (left) and detached resonance (right). The first, third, fifth and seventh harmonics and the total response are represented by red, blue, violet, magenta and black lines, respectively.

Figure 25 shows the amplitudes and the phases of the overall response, of the main harmonic and of the first superharmonic and the relative phase between the main harmonic and the first superharmonic in the frequency domain. Note that the solutions belonging to the detached resonance curve show a phase equal to $\pi / 2$, condition shared only with the solution of the main resonance frequency.
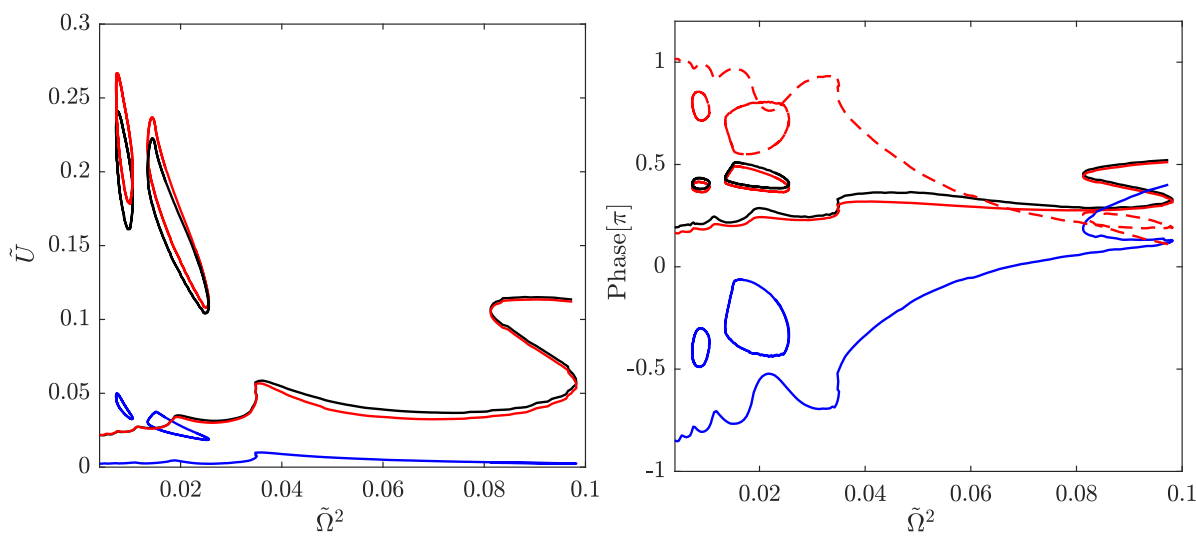

Fig. 25 Nondimensional displacement (left) and phase angle (right) vs. nondimensional frequency for the $S_{3}$ system with $\tilde{K}_{n}=1.2 \alpha, Y=Z_{m}, y_{s}=0.2$ and $\tilde{A}_{g}=0.01072$. Black lines show the amplitude and phase angle of the total response, red lines and blue lines represent the amplitude and phase angle of the main harmonic and of the first superharmonic of order 1:3, respectively. The phase angle between the main harmonic and the first superharmonic is reported in gray lines.

\subsection{Bifurcation scenarios and quasi-periodicity}

As mentioned above, for low frequencies and high base accelerations, the periodic response of the $S_{3}$ system with $\tilde{K}_{n}=1.2 \alpha$ undergoes a loss of stability. By restricting our analysis to 
the frequency range reported in Fig. 26, a rich sequence of bifurcations is found. Moving from low to high frequencies, the first encountered bifurcation is a Neimarck-Sacker or secondary Hopf bifurcation (A), signalled by a pair of Floquet multipliers crossing the unit circle away from the real axis (red circles). The solution emerging out of the Neimarck-Sacker bifurcation is a quasi-periodic solution. However, past the bifurcation the continuation of the unstable periodic solution cannot be successfully achieved. On the other hand, between $\mathrm{C}$ and $\mathrm{D}$ a stable branch of periodic solutions is found, which loses its stability at $\mathrm{C}$ due to a symmetry-breaking bifurcation. The two branches of mirror nonsimmetric periodic attractors lose their stability at B due to a period doubling bifurcation, circumstance signalled by the fact that one of the Floquet multipliers crosses the unit circle through -1 . In D the solution experiences a fold bifurcation, whereby one of the Floquet multipliers crosses the unit circle along the positive real axis. Finally, in E a new Neimarck-Sacker bifurcation is manifested and afterwards path following of the stationary solutions breaks down.
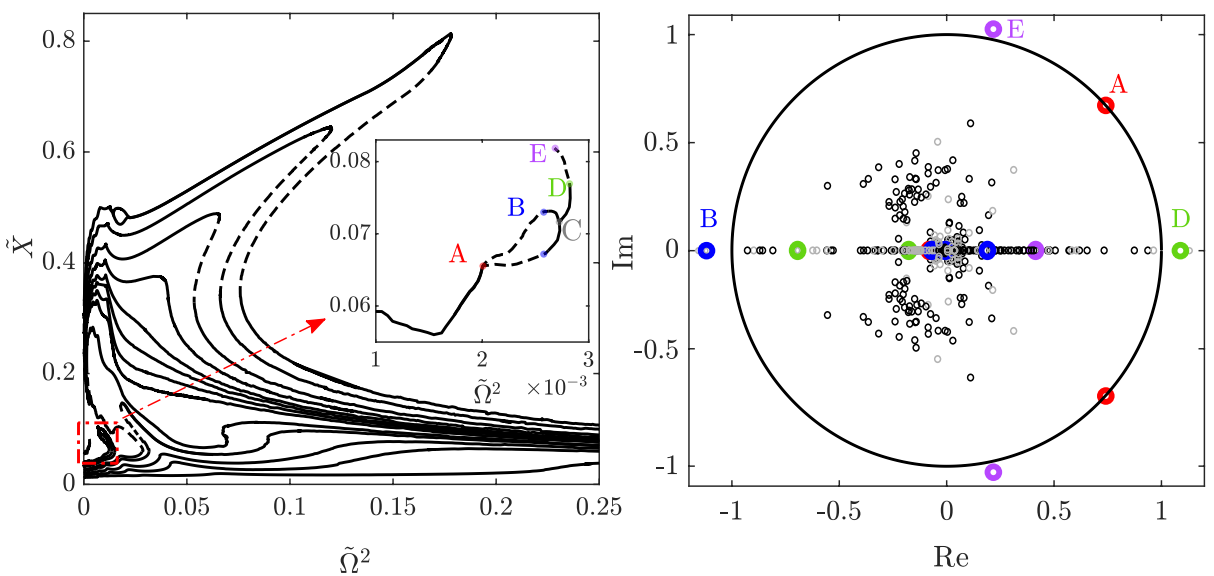

Fig. 26 FRCs for the $S_{3}$ system with $\tilde{K}_{n}=1.2 \alpha, Y=Z_{m}, y_{s}=0.5$ for a nondimensional ground acceleration equal to $\tilde{A}_{g}=0.0148$ (left) and imaginary parts vs. real parts of Floquet multipliers (right).

To investigate more in depth the scenario between the Neimarck-Sacker bifurcation in A and the period-doubling in $\mathrm{B}$, bifurcation diagrams were constructed by direct numerical integration of the equations of motion (see Fig. 27). The time step was fixed by dividing the excitation period into 4096 equally spaced points. The integration was carried out over 2,000 cycles of the excitation by considering as initial conditions those obtained at the previous excitation frequency and the last 64 points of the Poincare map were recorded. It is possible to note that in A the solution becomes quasiperiodic, through the mentioned secondary Hopf bifurcation, while from B to the left the solution becomes quasi-periodic by means of a more complex sequence of bifurcations. Figure 28 shows a symmetry breaking bifurcation at the frequency denoted by 2 , singled out by the birth of an even superharmonic component, after the limit cycle exhibited at 1 . This symmetry breaking paves the way to two distinct branches of nonsymmetric mirror solutions, whose orbits include the limit cycle and towards this they expand. In this frequency range the solution turns out to jump from one nonsymmetric branch to the other for any small frequency variation. Each of these branches undergoes a cascade of successive period-doubling bifurcation induced by the birth of a subharmonic component of order 2:1 at 3 and of subharmonics of order 4:1 and 2:1 at 4 . 


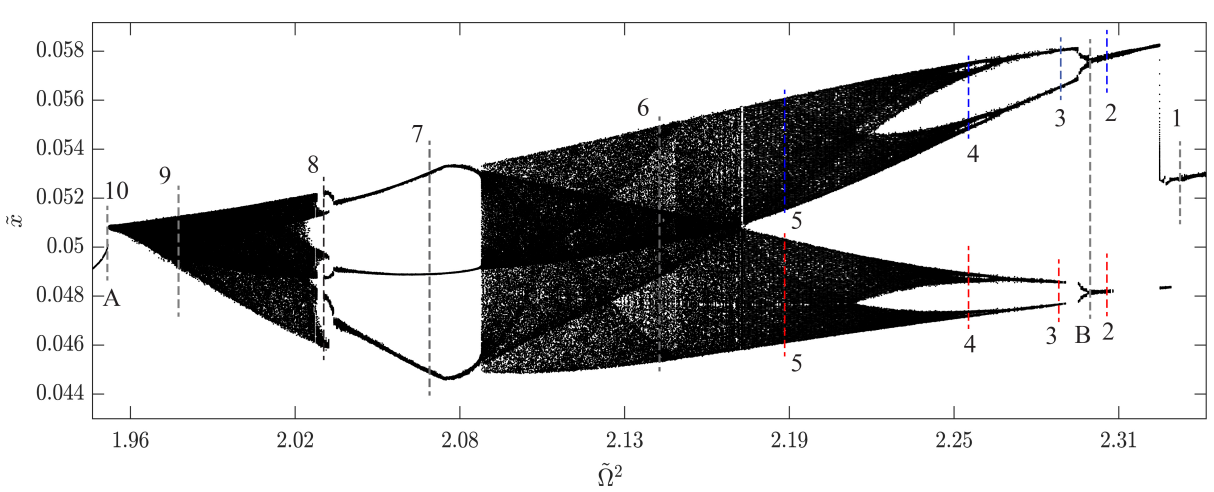

Fig. 27 Bifurcation diagram for the system with $\tilde{K}_{n}=1.2 \alpha, Y=Z_{m}, y_{s}=0.5$ for a nondimensional ground acceleration equal to $\tilde{A}_{g}=0.0148$.

This cascade of period-doubling bifurcations, giving rise to a nidification of subharmonics and superharmonics, may lead to a choatic attractor. When the orbits of the nonsymmetric solutions touch the orbit of the limit cycle there is a reverse symmetry breaking and the solution regains symmetry, signalled by the disappearance of the even superharmonic component (6). In the frequency range between 2.04 and 2.09, the ratio between the modulation frequency and the carrier frequency locks into a rational number, due to the so-called frequency-locking phenomenon with three-period motions, supported by the 3:1 subharmonic (7). Past this window, a new symmetry breaking is experienced by the 3-T solution, yielding two distinct branches of 3-T solutions in which, in addition to the 3:1 subharmonic, there appears an even superharmonic of order 1:2 (8). The symmetry breaking forces the solution to transition towards a quasiperiodic symmetric solution regime (9). Finally, the amplitude of the phase plane portion covered by the trajectory progressively gets reduced until 10, where a reverse secondary Hopf bifurcation makes the solution stable.

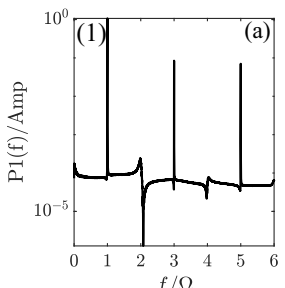

$f / \Omega$

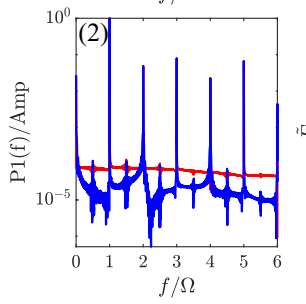

Fig. 28 FFTs of the response (a), force-displacement cycles (b), phase portraits (c) and Poincarè map (d) of the system when $\tilde{K}_{n}=1.2 \alpha, Y=Z_{m}, y_{s}=0.5$, the nondimensional ground acceleration is set to $\tilde{A}_{g}=0.0148$ for the frequencies corresponding to 1,2 . 

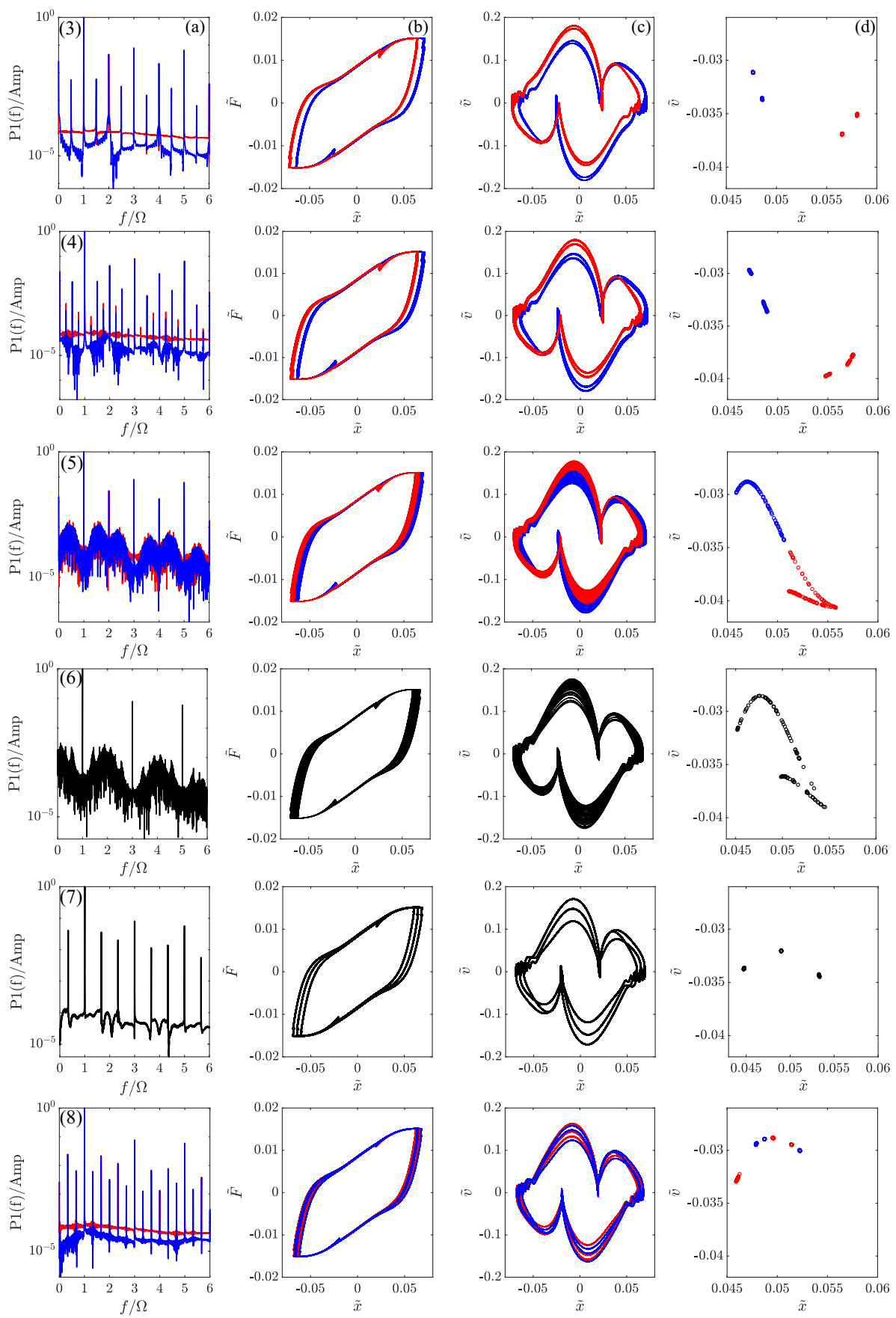

Fig. 29 FFTs of the response (a), force-displacement cycles (b), phase portraits (c) and Poincarè map (d) of the system when $\tilde{K}_{n}=1.2 \alpha, Y=Z_{m}, y_{s}=0.5$ for a nondimensional ground acceleration equal to $\tilde{A}_{g}=0.0148$ for the frequencies referred to as 3, 4, 5, 6, 7, 8 in Fig. 27. 

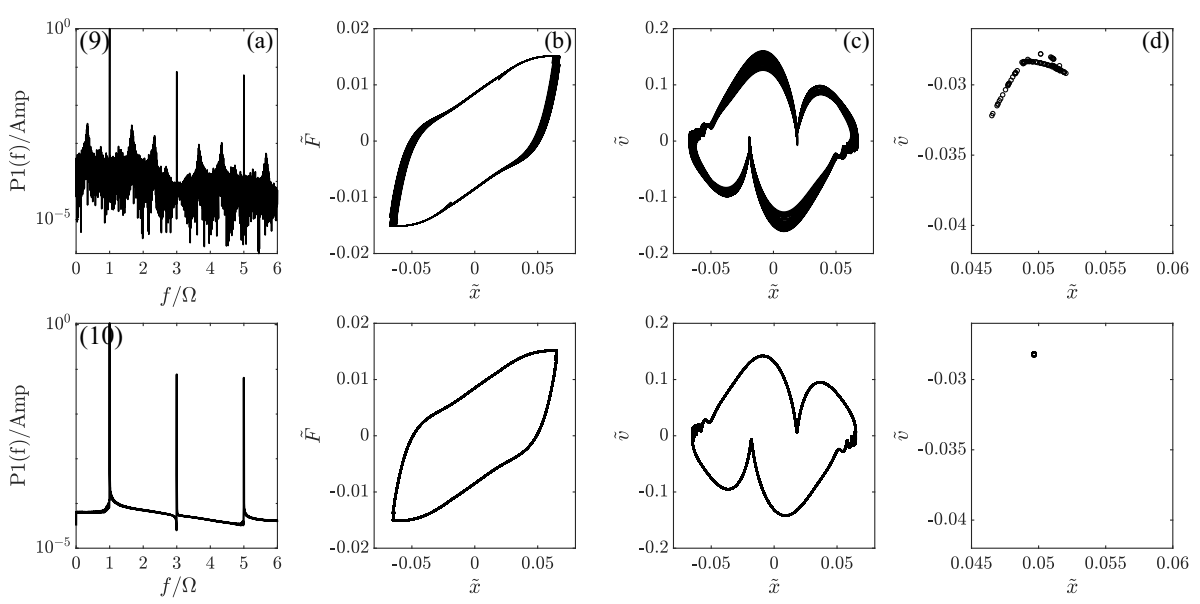

Fig. 30 FFTs of the response (a), force-displacement cycles (b), phase portraits (c) and Poincarè map (d) of the system when $\tilde{K}_{n}=1.2 \alpha, Y=Z_{m}, y_{s}=0.5$ for a nondimensional ground acceleration equal to $\tilde{A}_{g}=0.0148$ for the frequencies referred to as 9 and 10 in Fig. 27.

\subsubsection{Bifurcation scenarios for the tri-stable configuration}

For $\tilde{K}_{n}=1.2 \alpha$, the bifurcation scenario engages only ultra-low frequencies and a small displacements range. On the other hand, when $\tilde{K}_{n}>1.2 \alpha$, the bifurcation scenario involves much larger ranges of frequencies and displacements. As shown, for $\tilde{K}_{n}>1.2 \alpha$ the system is tri-stable and the presence of the two lateral attractors breaks the symmetry of the response. Because of the existence of symmetry breaking and period-doubling bifurcations, the response is quasi-periodic for most of the frequencies within the considered range. In order to obtain the FRCs of the tri-stable configuration (i.e., $\tilde{K}_{n}=1.4 \alpha$ ), the equations of motion are numerically integrated for a harmonic base excitation over 1,000 periods and the maximum amplitudes exhibited in the last 50 cycles, together with the Poincare sections, are recorded for each frequency within the range (see Fig. 31). Depending on the initial conditions, the mass can vibrate around the origin, the left or the right equilibrium positions. Regardless of the equilibrium around which the mass vibrates, the adjacent attractor breaks the symmetry of the response and leads to quasi-periodicity. Further, when the mass vibrates around one of the lateral equilibria, due to the stronger effects of the cubic stiffness, the acceleration transmissibility is higher compared with that exhibited by the mass vibrating around the origin. For limited frequency intervals, the phase-locking phenomenon is observable together with a reduction of transmissibility with respect to the adjacent quasi-periodic response.

By analysing more in depth the response in Fig. 31, we can note that for low frequencies $\left(0<\tilde{\Omega}^{2}<0.022\right)$, the system has sufficient energy to complete symmetric periodic cycles. For higher frequencies $\left(0.022<\tilde{\Omega}^{2}<0.042\right)$, because of a folding bifurcation, two different responses are exhibited by the system depending on the initial conditions. The high amplitude response, such as the response of the previous frequency range, exhibits stable cycles. On the other hand, the low amplitude solution, with a lower associated energy, suffers the attraction of the lateral equilibria and shows a quasi-periodic behavior. Increasing the frequency up to $\tilde{\Omega}^{2}=0.042$, a downward jump occurs together with the birth of two mirrored asymmetric solutions, each of which experiences cascades of period-doubling bifurcations. In the frequency range between $0.118<\tilde{\Omega}^{2}<0.18$, the existing solutions regain 
periodicity and two new mirrored asymmetric and quasi-periodic solutions appear along the lateral right and left equilibrium. Finally, when $\tilde{\Omega}^{2}=0.165$, the first two mirrored solutions coalesce into one symmetric periodic solution while the two lateral asymmetric solutions regain stability. In this latter frequency range, an amplification of both accelerations and displacements is observable.

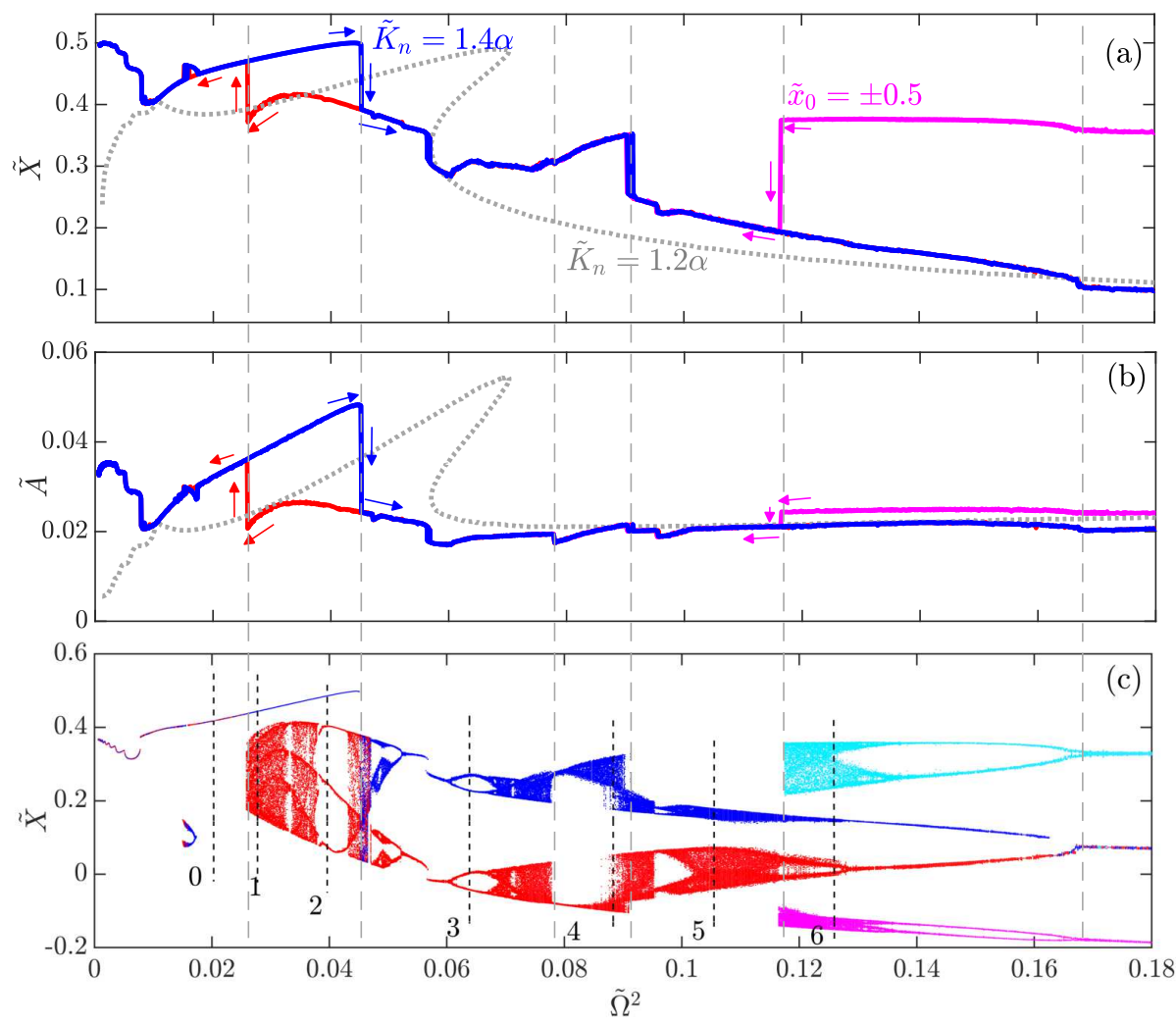

Fig. 31 FRCs in terms of nondimensional displacements (a) and accelerations (b) and bifurcation diagram (c) for the $S_{3}$ system with $\tilde{K}_{n}=1.4 \alpha, Y=Z_{m}, y_{s}=0.2$ for a ground acceleration equal to $\tilde{A}_{g}=0.0142$. Blue lines represent the responses obtained for the forward frequency sweep while red lines denote those obtained in reverse sweep. Magenta and cyan lines indicate the responses of the system with initial conditions $\tilde{x}_{0}=0.5$ and of $\tilde{x}_{0}=-0.5$, respectively. Finally, for comparative purposes, the responses of the mono-stable system with $\tilde{K}_{n}=1.2 \alpha$ are represented by gray lines.

In Fig. 32 the FFTs, hysteretic loops, phase portraits and Poincarè maps associated with the frequencies highlighted in Fig. 31 are reported. By focusing on the last section (6) the coexistence of four different types of response for the same excitation frequency is noted. The basins of attraction associated to this frequency are numerically obtained and reported in Fig. 33. 

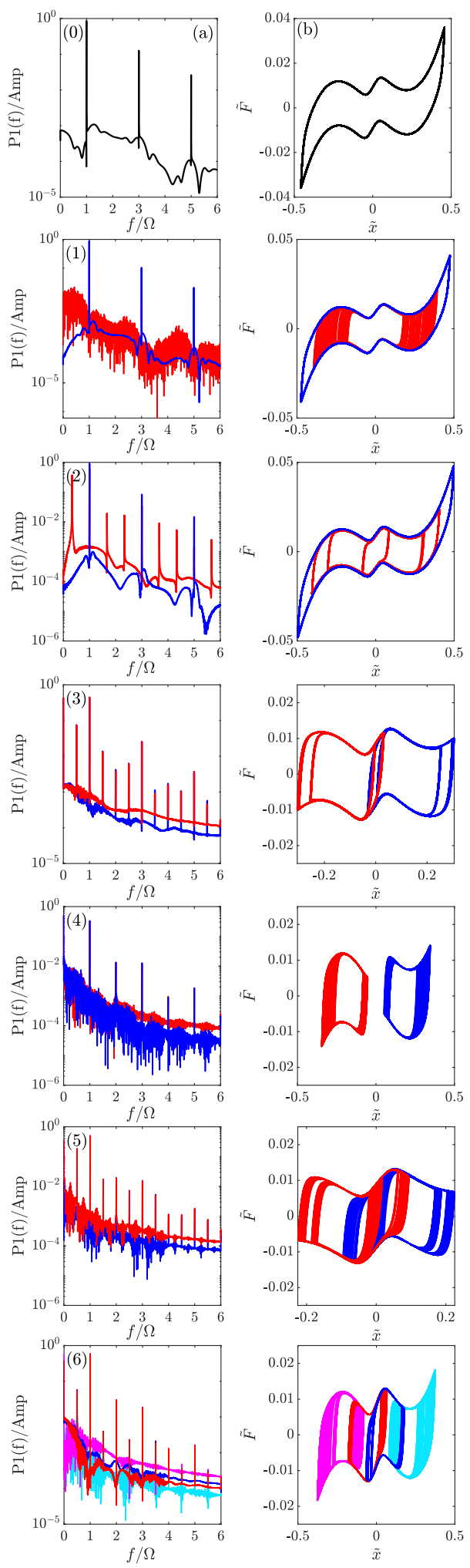
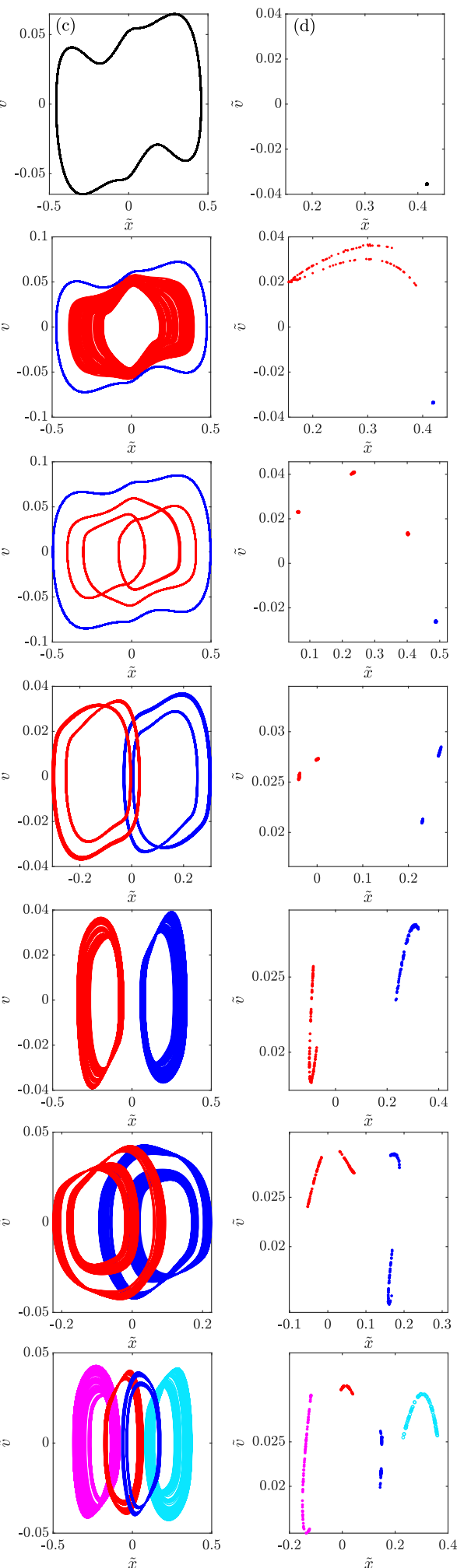

Fig. 32 FFTs of the response (a), force-displacement cycles (b), phase portraits (c) and Poincarè map (d) of the system when $\tilde{K}_{n}=1.4 \alpha, Y=Z_{m}, y_{s}=0.2$ and the nondimensional ground acceleration is set to $\tilde{A}_{g}=$ 0.0142 for the frequencies referred to as $0,1,2,3,4,5,6$ in Fig. 31. 
The coexistence of four different attractors, namely the left stable (LS) and unstable (LU) and the right stable (RS) and unstable (RU) equilibria, results in a high sensitivity of the dynamic response to the initial conditions, as manifested by the richness of the basins. From the progressive zooms of the basins of attraction (see Fig. 33c and Fig. 33d) it is notable that the strict adherence to the attractors succession order (LS, LU, RU, RS) leads to a fractal-like pattern.
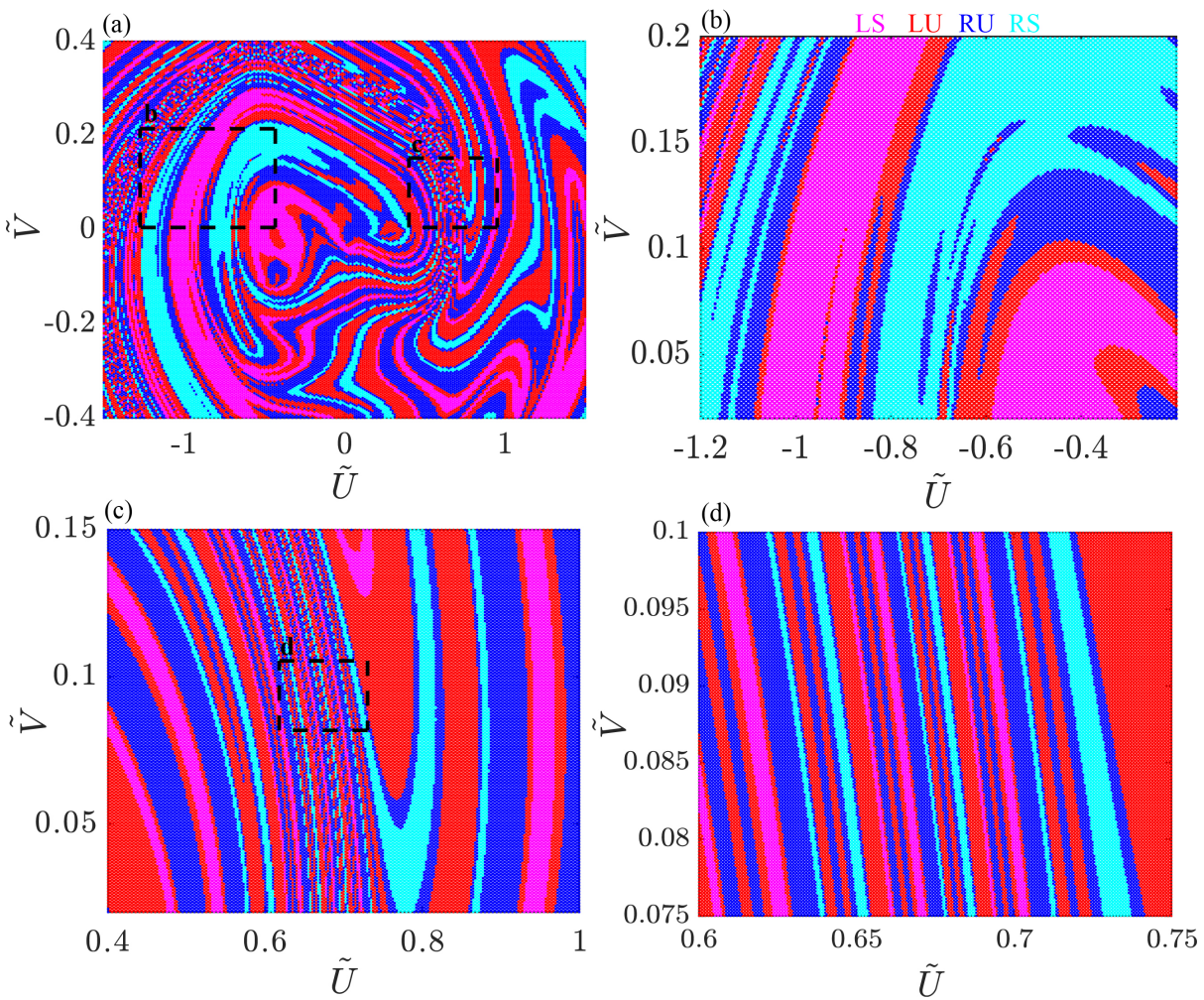

Fig. 33 (a) Basins of attraction for the system with $\tilde{K}_{n}=1.4 \alpha, Y=Z_{m}, y_{s}=0.2$ for a base acceleration $\tilde{A}_{g}=0.0142$ and frequency $\tilde{\Omega}^{2}=0.125$. Parts (b), (c) and (d) are zoomed-in regions bounded by the dashed rectangles. Magenta and red dots denote the initial conditions that lead to the left stable (LS) and unstable (LU) attractors, respectively, while, cyan and blue dots represent the initial conditions that lead to the right stable (RS) and unstable (RU) attractors, respectively.

\section{Conclusions}

A novel vibration isolation system featuring a negative stiffness mechanism and shape memory alloy nonlinear damping arranged in parallel with classical elastomeric hysteretic isolation devices is investigated parametrically for different levels of negative stiffness, SMA hysteretic damping ratio and yielding force.

The introduction of SMA alone, without negative stiffness, leads to a detrimental increase of the initial stiffness and, hence, to an increase of accelerations for low base excitations. On the 
other hand, by accurately tuning the negative stiffness with the SMA damping, a remarkable reduction of displacement and acceleration amplitudes can be achieved, while preserving a self-recentering capability without incurring an increase of acceleration transmissibility for low base excitations. To ensure an effective acceleration transmissibility reduction and, at the same time, the mono-stability and self-recentering capability of the isolated system, the optimum negative stiffness coefficient $\tilde{K}_{n}$ must be bounded within the range $\alpha<\tilde{K}_{n}<1.2 \alpha$, where $\alpha$ is the ratio between the post-elastic and the initial device stiffness. Regarding the SMA rheological element, the initial stiffness must be equal to the negative stiffness in order to keep the isolation system stiffness unaltered at the origin and, at the same time, develop a sufficiently high hysteretic damping. By considering $\tilde{K}_{n}=\alpha$, the optimum SMA element yielding force was found to be $\tilde{Y}=\alpha \tilde{x}_{g}$, where $\tilde{x}_{g}$ is the ratio between the gap displacement and the maximum allowable displacement. Moreover, a high hysteresis ratio $y_{s}$ was shown to entail a better isolation performance.

The study of the nonlinear dynamic response and its bifurcations has unfolded very rich bifurcation scenarios with detached resonances and unusual interactions between the primary resonance and superharmonic resonances, or between superharmonic resonances of various orders, featuring multiplicity of coexisiting attractors, secondary Hopf bifurcations leading to quasi-periodicity, synchronization, symmetry breaking and period-doubling cascades. The detached resonance curves and bifurcations were numerically explored for high levels of negative stiffness and their nonlinear impact on isolation performance was discussed. In particular, when $\tilde{K}_{n}=\alpha$ the peak of the detached resonance curve was found to be lower than the peak of the main resonance curve, thus not affecting the isolation performance. Instead, for a higher negative stiffness value (i.e., $\tilde{K}_{n}=1.2 \alpha$ ), the peak of the detached resonance curve was found to be larger than the primary resonance peak, hence, notably affecting the isolation performance. Moreover, the interesting quasi-periodicity of the response of the tri-stable configuration obtained for higher levels of negative stiffness (i.e., $\tilde{K}_{n}=1.4 \alpha$ ), and the subsequent dynamic amplification, were illustrated extensively. The obtained results pave the way towards a streamlined design process which aims to optimize the isolation performance of the proposed negative stiffness-SMA device in terms of transmissibility and dynamic stability.

\section{Declarations}

Acknowledgments

This work was partially supported by the PRIN Grant n. 2017L7X3CS - 002 (3D PRINTING: A BRIDGE TO THE FUTURE. Computational methods, innovative applications, experimental validations of new materials and technologies) and by the 2019 Sapienza Grant n. RG11916B8160BCCC (Vibration mitigation via advanced engineered devices and materials) which are gratefully acknowledged.

\section{Conflict of interest}

The authors have no conflicts of interest to declare that are relevant to the content of this article. 
Authors contributions

All authors contributed to the study conception and design. Material preparation, data collection and analysis were performed by Andrea Salvatore. The first draft of the manuscript was written by Andrea Salvatore and all authors commented on previous versions of the manuscript. All authors read and approved the final manuscript.

\section{References}

1. Pasala DTR, Sarlis AA, Nagarajaiah S, et al, Negative stiffness device for seismic response control of multistory buildings, Structures Congress, 2012

2. Taylor D, Nagarajaiah S, Reinhorn AM, et al, Adaptive negative stiffness: A new structural modification approach for seismic protection, Journal of Structural Engineering (139), 2013

3. Attary N, Symans M, Nagarajaiah S, Development of a rotation-based negative stiffness device for seismic protection of structures, Journal of Vibration and Control (23), 853-867, 2017

4. Iemura $\mathrm{H}$, Application of pseudo-negative stiffness control to the benchmark bridge, Journal of Structural Control (10-3), 187-203, 2003

5. Iemura H, Kouchiyama O, Toyooka A, Shimoda I, Development of the Friction-Based Passive Negative Stiffness Damper and Its Verification Tests Using Shaking Table, World Conference on Earthquake Engineering, 2008

6. Iemura $\mathrm{H}$, Pradono $\mathrm{MH}$, Advances in the development of pseudo-negative-stiffness dampers for seismic response control, Structural Control and Health Monitoring (16), 784-799, 2010

7. Pradono MH, Iemura $\mathrm{H}$, Passively controlled MR damper in the benchmark structural control problem for seismically excited highway bridge, Structural Control and Health Monitoring (16), 626-638, 2010

8. Tan X, Wang B, Chen S, et al, A novel cylindrical negative stiffness structure for shock isolation, Composite Structures (214), 397-405, 2019

9. Winterflood J, Blair DG, Slagmolen B, High performance vibration isolation using springs in Euler column buckling mode, Physics Letters A (300), 122-130, 2002

10. Platus DL. Negative stiffness mechanism vibration isolation systems, Proceedings Volume 1619, Vibration Control in Microelectronics, Optics, and Metrology, 1992

11. Alhan C, Gavin HP, Aldemir U, Optimal control: Basis for performance comparison of passive and semiactive isolation systems, Journal of Engineering Mechanics (132), 2006

12. Carrella A, Brennan MJ, Waters TP, Static analysis of a passive vibration isolator with quasi-zerostiffness characteristic, Journal of Sound and Vibration (301), 678-689, 2007

13. Kovacic I, Brennan MJ, Waters TP, A study of a nonlinear vibration isolator with a quasi-zero stiffness characteristic, Journal of Sound and Vibration (315), 700-711, 2008

14. Zhou N, Liu K, A tunable high-static-low-dynamic stiffness vibration isolator, Journal of Sound and Vibration (329), 1254-1273, 2010

15. Le TD, Ahn KK, A vibration isolation system in low frequency excitation region using negative stiffness structure for vehicle seat, Journal of Sound and Vibration (330), 6311-6335, 2011

16. Carrella A, Brennan MJ, Waters TP, Lopes V, Force and displacement transmissibility of a nonlinear isolator with high-static-low-dynamic-stiffness, International Journal of Mechanical Sciences (55), 22-29, 2012

17. Yang J, Xiong YP, Xing JT, Dynamics and power flow behaviour of a nonlinear vibration isolation system with a negative stiffness mechanism, Journal of Sound and Vibration (332), 167-183, 2013

18. Huang X, Liu X, Sun J, et al, Vibration isolation characteristics of a nonlinear isolator using euler buckled beam as negative stiffness corrector: A theoretical and experimental study, Journal of Sound and Vibration (333), 1132-1148, 2014

19. Hao Z, Cao Q, The isolation characteristics of an archetypal dynamical model with stable-quasi-zerostiffness, Journal of Sound and Vibration (340), 61-79, 2015

20. Zheng Y, Zhang X, Luo Y, et al, Design and experiment of a high-static-low-dynamic stiffness isolator using a negative stiffness magnetic spring, Journal of Sound and Vibration (360), 31-52, 2016

21. Lu ZQ, Brennan M, Ding H, Chen LQ, High-static-low-dynamic-stiffness vibration isolation enhanced by damping nonlinearity, Science China Technological Sciences (62), 1103-1110, 2018

22. Cimellaro GP, Domaneschi M, Warn G, Three-Dimensional Base Isolation Using Vertical Negative Stiffness Devices, Journal of Earthquake Engineering (24), 2004-2032, 2018

23. Wang X, Liu H, Chen Y, Gao P, Beneficial stiffness design of a high-static-low-dynamic-stiffness vibration isolator based on static and dynamic analysis, International Journal of Mechanical Sciences (142), $235-244,2018$ 
24. Zhou Y, Chen P, Mosqueda G, Analytical and Numerical Investigation of Quasi-Zero Stiffness Vertical Isolation System, Journal of Engineering Mechanics (145), 2019

25. Sun M, Song G, Li Y, Huang Z, Effect of negative stiffness mechanism in a vibration isolator with asymmetric and high-static-low-dynamic stiffness, Mechanical Systems and Signal Processing (124), 388407, 2019

26. Shi X, Zhu S, A comparative study of vibration isolation performance using negative stiffness and inerter dampers, Journal of the Franklin Institute (356), 7922-7946, 2019

27. Yan B, Ma H, Jian B, Wang K, Wu C, Nonlinear dynamics analysis of a bi-state nonlinear vibration isolator with symmetric permanent magnets, Nonlinear Dynamics (97), 2499-2519, 2019

28. Bian J, Jing X, Analysis and design of a novel and compact X-structured vibration isolation mount (XMount) with wider quasi-zero-stiffness range, Nonlinear Dynamics (101), 2195-2222, 2020

29. Gao X, Teng HD, Dynamics and isolation properties for a pneumatic near-zero frequency vibration isolator with nonlinear stiffness and damping, Nonlinear Dynamics (102), 2205-2227, 2020

30. Liu C, Yu K, Accurate modeling and analysis of a typical nonlinear vibration isolator with quasi-zero stiffness, Nonlinear Dynamics (100), 2141-2165, 2020

31. Sonfack Bouna H, Nana Nbendjo BR, Woafo P, Isolation performance of a quasi-zero stiffness isolator in vibration isolation of a multi-span continuous beam bridge under pier base vibrating excitation, Nonlinear Dynamics (100), 1125-1141, 2020

32. Wang K, Zhou J, Chang Y, Ouyang $\mathrm{H}$, Xu D, A nonlinear ultra-low-frequency vibration isolator with dual quasi-zero-stiffness mechanism, Nonlinear Dynamics (101), 755-773, 2020

33. Donmez A, Cigeroglu E, Ozgen OG, An improved quasi-zero stiffness vibration isolation system utilizing dry friction damping, Nonlinear Dynamics (101), 107-121, 2020

34. Liu M, Zhou P, Li H, Novel Self-Centering Negative Stiffness Damper Based on Combination of Shape Memory Alloy and Prepressed Springs, Journal of Aerospace Engineering (31), 2018

35. Bouc R, Forced vibration of mechanical systems with hysteresis, Materials Science 1967

36. Wen Y, Method for random vibration of hysteretic Systems, Journal of the Engineering Mechanics Division (102), 249-263, 1976

37. Charalampakis AE, Tsiatas GC, A Simple Rate-Independent Uniaxial Shape Memory Alloy (SMA) Model, Frontiers in Built Environment (4), 2018

38. Carboni B, Lacarbonara W, Dynamic response of nonlinear oscillators with hysteresis, Proceedings of the ASME 2015 IDETC-CIE conference (6), 2015

39. Lacarbonara $\mathrm{W}$, Vestroni $\mathrm{F}$, and Capecchi $\mathrm{D}$, Poincarè map-based continuation of periodic orbits in dynamic discontinuous and hysteretic systems, 17th Biennial ASME Conference on Mechanical Vibration and Noise, 1999

40. Habib G, Cirillo GI, Kerschenc G, Uncovering detached resonance curves in single-degree-of-freedom systems, Procedia Engineering (199), 649-656, 2017

41. Gatti G, Brennan MJ, Kovacic I, On the interaction of the responses at the resonance frequencies of a nonlinear two degrees-of-freedom system, Physica D: Nonlinear Phenomena (239), 591-599, 2016

42. Formica G, Lacarbonara W, Asymptotic dynamic modeling and response of hysteretic nanostructured beams, Nonlinear Dynamics (99), 227-248, 2020 